\title{
OBSERVABILITY PROPERTIES OF A SEMI-DISCRETE 1D WAVE EQUATION DERIVED FROM A MIXED FINITE ELEMENT METHOD ON NONUNIFORM MESHES
}

\author{
SYLVAIN ERVEDOZA ${ }^{1}$
}

\begin{abstract}
The goal of this article is to analyze the observability properties for a space semi-discrete approximation scheme derived from a mixed finite element method of the $1 \mathrm{~d}$ wave equation on nonuniform meshes. More precisely, we prove that observability properties hold uniformly with respect to the mesh-size under some assumptions, which, roughly, measures the lack of uniformity of the meshes, thus extending the work [Castro and Micu, Numer. Math. 102 (2006) 413-462] to nonuniform meshes. Our results are based on a precise description of the spectrum of the discrete approximation schemes on nonuniform meshes, and the use of Ingham's inequality. We also mention applications to the boundary null controllability of the $1 \mathrm{~d}$ wave equation, and to stabilization properties for the $1 \mathrm{~d}$ wave equation. We finally present some applications for the corresponding fully discrete schemes, based on recent articles by the author.
\end{abstract}

Mathematics Subject Classification. 35L05, 35P20, 47A75, 93B05, 93B07, 93B60, 93D15.

Received May 25, 2008. Revised September 30, 2008.

Published online December 19, 2008.

\section{INTRODUCTION}

The goal of this article is to address the observability properties for a semi-discrete 1d wave equation. We consider the following $1 \mathrm{~d}$ wave equation:

$$
\begin{cases}\partial_{t t}^{2} u-\partial_{x x}^{2} u=0, & (x, t) \in(0,1) \times \mathbb{R}, \\ u(0, t)=u(1, t)=0, & t \in \mathbb{R}, \\ u(x, 0)=u^{0}(x), \partial_{t} u(x, 0)=u^{1}(x), & x \in(0,1),\end{cases}
$$

where $u^{0} \in H_{0}^{1}(0,1)$ and $u^{1}(x) \in L^{2}(0,1)$. The energy of solutions of (1.1), given by

$$
E(t)=\frac{1}{2} \int_{0}^{1}\left|\partial_{t} u(t, x)\right|^{2}+\left|\partial_{x} u(t, x)\right|^{2} \mathrm{~d} x,
$$

is constant.

Keywords and phrases. Spectrum, observability, wave equation, semi-discrete systems, controllability, stabilization.

1 Laboratoire de Mathématiques de Versailles, Université de Versailles Saint-Quentin, 45 avenue des États-Unis, 78035 Versailles Cedex, France. sylvain.ervedoza@math.uvsq.fr 
It is well-known (see [21]) that for all $T>0$, there exists a constant $K_{T}$ such that the admissibility inequality

$$
\int_{0}^{T}\left|\partial_{x} u(0, t)\right|^{2} \mathrm{~d} t \leq K_{T} E(0)
$$

holds for any solution of (1.1) with $\left(u^{0}, u^{1}\right) \in H_{0}^{1}(0,1) \times L^{2}(0,1)$.

Besides, for any time $T \geq 2$, there exists a positive constant $k_{T}$ such that the boundary observability inequality

$$
k_{T} E(0) \leq \int_{0}^{T}\left|\partial_{x} u(0, t)\right|^{2} \mathrm{~d} t
$$

holds for any solution of (1.1) with $\left(u^{0}, u^{1}\right) \in H_{0}^{1}(0,1) \times L^{2}(0,1)$.

Inequalities (1.3)-(1.4) arise naturally when dealing with boundary controllability properties of the $1 \mathrm{~d}$ wave equation, see [21]. Indeed, the observability and controllability properties are dual notions. We will clarify this relation in Section 3.

Let us also present another relevant observability inequality, which is useful when dealing with distributed controls or stabilization properties of damped wave equations (see [16,21]). If $(a, b)$ denotes a non empty subinterval of $(0,1)$, the following distributed observability property holds: for any time $T>2 \max \{a, 1-b\}$, there exists a constant $C_{1}$ such that any solution of (1.1) with initial data $\left(u^{0}, u^{1}\right) \in H_{0}^{1}(0,1) \times L^{2}(0,1)$ satisfies:

$$
E(0) \leq C_{1} \int_{0}^{T} \int_{a}^{b}\left|\partial_{t} u(x, t)\right|^{2} \mathrm{~d} x \mathrm{~d} t .
$$

In the sequel, we will consider observability properties for the $1 \mathrm{~d}$ space semi-discrete wave equation derived from a mixed finite element method on a nonuniform mesh.

For any integer $n \in \mathbb{N}^{*}$, let us consider a mesh $\mathcal{S}_{n}$ given by $n+2$ points as:

$$
0=x_{0, n}<x_{1, n}<\ldots<x_{n, n}<x_{n+1, n}=1, \quad h_{j+1 / 2, n}=x_{j+1, n}-x_{j, n}, \quad j \in\{0, \ldots, n\} .
$$

On $\mathcal{S}_{n}$, the mixed finite element approximation scheme for system (1.1) reads as (see [7,15] or [5]):

$$
\left\{\begin{array}{c}
\frac{h_{j-1 / 2, n}}{4}\left(u_{j-1, n}^{\prime \prime}+u_{j, n}^{\prime \prime}\right)+\frac{h_{j+1 / 2, n}}{4}\left(u_{j, n}^{\prime \prime}+u_{j+1, n}^{\prime \prime}\right) \\
=\frac{u_{j+1, n}-u_{j, n}}{h_{j+1 / 2, n}}-\frac{u_{j, n}-u_{j-1, n}}{h_{j-1 / 2, n}}, \quad j=1, \ldots, n, t \in \mathbb{R}, \\
u_{0, n}(t)=u_{n+1, n}(t)=0, \quad t \in \mathbb{R}, \\
u_{j, n}(0)=u_{j, n}^{0}, \quad u_{j, n}^{\prime}(0)=u_{j, n}^{1}, \quad j=1, \ldots, n .
\end{array}\right.
$$

The notations we use are the standard ones: A prime denotes differentiation with respect to time, and $u_{j, n}(t)$ is an approximation of the solution $u$ of (1.1) at the point $x_{j, n}$ at time $t$.

System (1.7) is conservative. The energy of solutions $u_{n}$ of (1.7), given by

$$
E_{n}(t)=\frac{1}{2} \sum_{j=0}^{n} h_{j+1 / 2, n}\left(\frac{u_{j+1, n}(t)-u_{j, n}(t)}{h_{j+1 / 2, n}}\right)^{2}+\frac{1}{2} \sum_{j=0}^{n} h_{j+1 / 2, n}\left(\frac{u_{j+1, n}^{\prime}(t)+u_{j, n}^{\prime}(t)}{2}\right)^{2}, \quad t \in \mathbb{R},
$$

is constant.

In this semi-discrete setting, we will investigate the observability properties corresponding to (1.4) and (1.5), and especially under which assumptions on the meshes $\mathcal{S}_{n}$ we can guarantee discrete observability inequalities to be uniform with respect to $n$. 
For this purpose, we introduce the notion of regularity of a mesh:

Definition 1.1. For a mesh $\mathcal{S}_{n}$ given by $n+2$ points as in (1.6), we define the regularity of the mesh $\mathcal{S}_{n}$ by

$$
\operatorname{Reg}\left(\mathcal{S}_{n}\right)=\frac{\max _{j}\left\{h_{j+1 / 2, n}\right\}}{\min _{j}\left\{h_{j+1 / 2, n}\right\}} .
$$

Given $M \geq 1$, we say that a mesh $\mathcal{S}_{n}$ given by $n+2$ points as in (1.6) is $M$-regular if

$$
\operatorname{Reg}\left(\mathcal{S}_{n}\right)=\frac{\max _{j}\left\{h_{j+1 / 2, n}\right\}}{\min _{j}\left\{h_{j+1 / 2, n}\right\}} \leq M
$$

Obviously, a 1-regular mesh is uniform. In other words, the regularity of the mesh $\operatorname{Reg}\left(\mathcal{S}_{n}\right)$ measures the lack of uniformity of the mesh.

Within the class of $M$ regular meshes, we will prove the following observability properties:

Theorem 1.2. Let $M$ be a real number greater than one, and consider a sequence $\left(\mathcal{S}_{n}\right)_{n}$ of $M$-regular meshes.

Then for any time $T>2$, there exist positive constants $k_{T}$ and $K_{T}$ such that for all integer $n$, any solution $u_{n}$ of (1.7) satisfies

$$
k_{T} E_{n}(0) \leq \int_{0}^{T}\left(\left|\frac{u_{1, n}(t)}{h_{1 / 2, n}}\right|^{2}+\left|u_{1, n}^{\prime}(t)\right|^{2}\right) \mathrm{d} t \leq K_{T} E_{n}(0) .
$$

Besides, if $J=(a, b) \subset(0,1)$ denotes a subinterval of $(0,1)$, then, for any time $T>2$, there exists a constant $C_{1}$ such that for all integer $n$, any solution $u_{n}$ of (1.7) satisfies

$$
E_{n}(0) \leq C_{1} \int_{0}^{T} \sum_{x_{j, n} \in J} h_{j+1 / 2, n}\left(\frac{u_{j, n}^{\prime}(t)+u_{j+1, n}^{\prime}(t)}{2}\right)^{2} \mathrm{~d} t .
$$

Obviously, these properties are discrete versions of inequalities (1.3), (1.4) and (1.5). Also note that the right hand-side inequality in (1.11) holds, as (1.3), for all time $T>0$, taking $K_{T}=K_{3}$ for $T \leq 2$.

Theorem 1.2 is based on an explicit spectral analysis of (1.7) in the discrete setting, that proves the existence of a gap between the eigenvalues of the space discrete operator in (1.7). Thanks to Ingham's inequality [18], this reduces the analysis to the study of the observability properties of the eigenvectors of (1.7), which will again be deduced from the explicit form of the spectrum of (1.7).

Besides, we emphasize that Theorem 1.2 provides uniform (with respect to $n$ ) observability results. Therefore, as in the continuous setting, Theorem 1.2 has several applications to controllability and stabilization properties for the space semi-discrete $1 \mathrm{~d}$ wave equations (1.7). In Section 3, similarly as in [5], using precisely the same duality as in the continuous case, we present an application to the boundary null controllability of the space semi-discrete approximation scheme of the 1d wave equation. Later, in Section 4, following [1], we study the decay properties of the energy for semi-discrete approximation schemes of $1 \mathrm{~d}$ damped wave equations.

In Section 5, we explain how Theorem 1.2 can be combined with the results in [11,12] to obtain observability and stabilization results for the corresponding fully discrete approximation schemes. Indeed, in the recent work [12], admissibility and observability properties for time-discrete approximation schemes of linear conservative systems were studied in a very general abstract setting. The approach developed in [12] allows to derive uniform observability inequalities for time-discrete approximation schemes in a systematic way. One of the interesting features of this technique is that it can be applied to fully discrete schemes as soon as the space semi-discrete approximation schemes satisfy uniform observability properties (see [12], Sect. 5). The study presented here fits in this abstract setting. Therefore, combining Theorem 1.2 and the results in [12], one can derive uniform (with respect to both time and space discretization parameters) observability properties for time-discrete approximation schemes of the space semi-discrete approximation schemes (1.7).

Later on, in [11], stabilization properties for time-discrete approximation schemes of abstract damped systems have been studied. In particular, in [11], several time-discrete approximation schemes have been designed 
to guarantee uniform (with respect to the time discretization parameter) stabilization properties, by adding a numerical viscosity term in time which efficiently damps out the spurious high frequency components created by the time discretization process. Besides, this can also be applied to families of uniformly exponentially stable systems, and in particular to families of space semi-discrete approximation schemes that fit into the abstract setting of [11], which is the case for discrete approximations of damped wave equations. Thus, using the results in [11], we will explain how to design fully discrete approximation schemes which are uniformly (with respect to both time and space discretization parameters) exponentially stable.

Let us briefly comment some relative works. Similar problems have been extensively studied in the last decade for various space semi-discrete approximation schemes of the $1 \mathrm{~d}$ wave equation, see for instance the review article [32]. The numerical schemes on uniform meshes provided by finite difference and finite element methods do not have uniform observability properties, whatever the time $T$ is (see [17]). This is due to high frequency waves that do not propagate, see $[22,29]$. To be more precise, these numerical schemes create some spurious high-frequency wave solutions that are localized.

However some remedies exist. The most natural one consists in filtering the initial data and thus removing these spurious waves, as in [17,31]. Another way to filter is to use the bi-grid method as introduced and developed in [14] and analyzed in [24]. A new approach was proposed recently in [25] based on wavelet filtering techniques. Let us also mention the results [10,26-28] that amounts to adding an extra term in (1.12) which is non-negligible only for the high frequencies. A last possible cure was proposed in [1,15] and later analyzed in [5]: a 1d semi-discrete scheme derived from a mixed finite element method was proposed, which has the property that the group velocity of the waves is bounded from below. Also note that an extension of [5] to the 2d case in the square was proposed in [6].

To the best of our knowledge, there is no result at all for the space semi-discrete wave equation on nonuniform meshes, although most of the domains used in practice are recovered by non periodic triangulations. A first step in this direction can be found in [26], in which a study of a non homogeneous string equation on a uniform mesh was proposed. This can indeed be seen, up to a change of variable, as a discretization of a wave equation with constant velocity on a slightly nonuniform mesh.

Let us also mention that some results are available in the context of the heat equation for space semi-discrete approximation schemes on nonuniform meshes in [19], even in dimension greater than 1 .

The outline of this paper is as follows. In Section 2, we precisely describe the spectrum of the space semidiscrete operator and prove Theorem 1.2. Sections 3 and 4 respectively aim at presenting precise applications of Theorem 1.2 to controllability and stabilization properties. Section 5 presents applications of Theorem 1.2 in the fully discrete setting for both observability and stabilization issues.

\section{SPECTRAL THEORY}

In this section, we first study the spectrum of the space semi-discrete operator in (1.7) on a general mesh $\mathcal{S}_{n}$ given by $n+2$ points as in (1.6). Second, we derive more precise estimates on the spectrum when $\mathcal{S}_{n}$ is an $M$-regular mesh. Third, we derive Theorem 1.2 from our analysis. Finally, we discuss the assumption on the regularity of the meshes, and show that, in some sense, the $M$-regularity assumption is sharp with respect to the observability properties given in Theorem 1.2.

Given a mesh $\mathcal{S}_{n}$ of $n+2$ points as in (1.6), since the system (1.7) is conservative, the spectral problem for (1.7) reads as: Find $\lambda_{n} \in \mathbb{R}$ and a non-trivial solution $\phi_{n}$ such that

$$
\left\{\begin{array}{l}
-\frac{\lambda_{n}^{2}}{4}\left(h_{j-1 / 2, n}\left(\phi_{j, n}+\phi_{j-1, n}\right)+h_{j+1 / 2, n}\left(\phi_{j, n}+\phi_{j+1, n}\right)\right) \\
=\frac{\phi_{j+1, n}-\phi_{j, n}}{h_{j+1 / 2, n}}-\frac{\phi_{j, n}-\phi_{j-1, n}}{h_{j-1 / 2, n}}, \quad j=1, \ldots, n, \\
\phi_{0, n}=\phi_{n+1, n}=0 .
\end{array}\right.
$$




\subsection{Computations of the eigenvalues for a general mesh}

In this subsection, we consider a general mesh $\mathcal{S}_{n}$ given by $n+2$ points as in (1.6).

Theorem 2.1. The spectrum of system (1.7) is precisely the set of $\pm \lambda_{n}^{k}$ with $k \in\{1, \ldots, n\}$, where $\lambda_{n}^{k}$ is defined by the implicit formula

$$
\sum_{j=0}^{n} \arctan \left(\frac{\lambda_{n}^{k} h_{j+1 / 2, n}}{2}\right)=\frac{k \pi}{2}
$$

The gap between two eigenvalues is bounded from below:

$$
\min _{k \in\{1, \ldots, n-1\}}\left\{\lambda_{n}^{k+1}-\lambda_{n}^{k}\right\} \geq \pi .
$$

Besides, for each $k \in\{1, \ldots, n\}$, the following estimate holds:

$$
\lambda_{n}^{k} \geq \lambda_{* n}^{k}=2(n+1) \tan \left(\frac{k}{n+1} \frac{\pi}{2}\right) \geq k \pi .
$$

Remark 2.2. Note that $\lambda_{* n}^{k}$ coincides with the $k$-th eigenvalue of system (1.7) for a uniform mesh constituted by $n+2$ points. Also note that $k \pi$ is the $k$-th eigenvalue of system (1.1). In other words, inequality (2.4) implies that the dispersion diagrams corresponding to the spectrum of (1.7) for a general nonuniform mesh, for a uniform mesh, and for the continuous system (1.1) are sorted.

Proof. To simplify notation, we drop the subscript $n$.

Let us introduce functions $p$ and $q$ corresponding to $\partial_{x} \phi$ and $i \lambda \phi$ in the continuous case:

$$
p_{j+1 / 2}=\frac{\phi_{j+1}-\phi_{j}}{h_{j+1 / 2}}, \quad q_{j+1 / 2}=\frac{i \lambda}{2}\left(\phi_{j}+\phi_{j+1}\right), \quad j \in\{0, \ldots, n\} .
$$

The spectral system (2.1) then becomes:

$$
\left\{\begin{array}{l}
\frac{i \lambda}{2}\left(h_{j-1 / 2} q_{j-1 / 2}+h_{j+1 / 2} q_{j+1 / 2}\right)=p_{j+1 / 2}-p_{j-1 / 2}, \quad j=1, \ldots, n, \\
\frac{i \lambda}{2}\left(h_{j-1 / 2} p_{j-1 / 2}+h_{j+1 / 2} p_{j+1 / 2}\right)=q_{j+1 / 2}-q_{j-1 / 2}, \quad j=1, \ldots, n,
\end{array}\right.
$$

with boundary conditions

$$
\frac{i \lambda h_{n+1 / 2}}{2} p_{n+1 / 2}+q_{n+1 / 2}=0, \quad \frac{i \lambda h_{1 / 2}}{2} p_{1 / 2}-q_{1 / 2}=0 .
$$

Equations (2.6) rewrite, for $j \in\{1, \ldots, n\}$, as:

$$
\left\{\begin{array}{l}
\left(\frac{i \lambda h_{j-1 / 2}}{2} q_{j-1 / 2}+p_{j-1 / 2}\right)+\left(\frac{i \lambda h_{j+1 / 2}}{2} q_{j+1 / 2}-p_{j+1 / 2}\right)=0 \\
\left(\frac{i \lambda h_{j-1 / 2}}{2} p_{j-1 / 2}+q_{j-1 / 2}\right)+\left(\frac{i \lambda h_{j+1 / 2}}{2} p_{j+1 / 2}-q_{j+1 / 2}\right)=0
\end{array}\right.
$$

For $j \in\{1, \ldots, n\}$, this leads to:

$$
\begin{aligned}
& \left(1+\frac{i \lambda h_{j-1 / 2}}{2}\right)\left(p_{j-1 / 2}+q_{j-1 / 2}\right)=\left(1-\frac{i \lambda h_{j+1 / 2}}{2}\right)\left(p_{j+1 / 2}+q_{j+1 / 2}\right) \\
& \left(1-\frac{i \lambda h_{j-1 / 2}}{2}\right)\left(p_{j-1 / 2}-q_{j-1 / 2}\right)=\left(1+\frac{i \lambda h_{j+1 / 2}}{2}\right)\left(p_{j+1 / 2}-q_{j+1 / 2}\right) .
\end{aligned}
$$


These two equations can be seen as propagation formulas, each term corresponding to $\partial_{t} w \pm \partial_{x} w$. Especially, they imply:

$$
\begin{aligned}
& p_{j+1 / 2}+q_{j+1 / 2}=\left(p_{1 / 2}+q_{1 / 2}\right)\left(\frac{2+i \lambda h_{1 / 2}}{2-i \lambda h_{j+1 / 2}}\right) \prod_{k=1}^{j-1}\left(\frac{2+i \lambda h_{k+1 / 2}}{2-i \lambda h_{k+1 / 2}}\right), \\
& p_{j+1 / 2}-q_{j+1 / 2}=\left(p_{1 / 2}-q_{1 / 2}\right)\left(\frac{2-i \lambda h_{1 / 2}}{2+i \lambda h_{j+1 / 2}}\right) \prod_{k=1}^{j-1}\left(\frac{2-i \lambda h_{k+1 / 2}}{2+i \lambda h_{k+1 / 2}}\right) .
\end{aligned}
$$

We remark that each term in the product has modulus 1 , and therefore there exists $\alpha_{j+1 / 2} \in(-\pi, \pi]$, given by $\tan \left(\alpha_{j+1 / 2} / 2\right)=\lambda h_{j+1 / 2} / 2$, such that:

$$
\frac{2+i \lambda h_{j+1 / 2}}{2-i \lambda h_{j+1 / 2}}=\exp \left(i \alpha_{j+1 / 2}\right)
$$

We also denote by $\beta_{j}$ the coefficient

which satisfies

$$
\beta_{j}=\frac{2+i \lambda h_{1 / 2}}{2-i \lambda h_{j+1 / 2}}
$$

$$
\frac{\beta_{j}}{\bar{\beta}_{j}}=\exp \left(i \alpha_{j+1 / 2}\right) \exp \left(i \alpha_{1 / 2}\right) \text {. }
$$

Combined with the boundary conditions, identities (2.8)-(2.9) give:

$$
\begin{aligned}
& p_{n+1 / 2}\left(1-\frac{i \lambda h_{n+1 / 2}}{2}\right)=\beta_{n} \exp \left(i \sum_{k=1}^{n-1} \alpha_{k+1 / 2}\right) p_{1 / 2}\left(1+\frac{i \lambda h_{1 / 2}}{2}\right) \\
& p_{n+1 / 2}\left(1+\frac{i \lambda h_{n+1 / 2}}{2}\right)=\bar{\beta}_{n} \exp \left(-i \sum_{k=1}^{n-1} \alpha_{k+1 / 2}\right) p_{1 / 2}\left(1-\frac{i \lambda h_{1 / 2}}{2}\right) .
\end{aligned}
$$

Then, if $\lambda$ is an eigenvalue, $\lambda$ satisfies:

$$
\left(\frac{\beta_{n}}{\bar{\beta}_{n}}\right)^{2} \exp \left(2 i \sum_{k=1}^{n-1} \alpha_{k+1 / 2}\right)=\exp \left(2 i \sum_{k=0}^{n} \alpha_{k+1 / 2}\right)=1 .
$$

To simplify notation, we define:

$$
f(\lambda)=4 \sum_{k=0}^{n} \arctan \left(\frac{\lambda h_{k+1 / 2}}{2}\right) .
$$

Due to (2.10), if $\lambda$ is an eigenvalue, there exists an integer $k$ such that:

$$
f(\lambda)=2 k \pi .
$$

The image of $f$ is exactly $(-2(n+1) \pi, 2(n+1) \pi)$, and therefore $k$ must belong to $\{-n, \ldots, n\}$.

Conversely, if $\lambda$ is a solution of $f(\lambda)=2 k \pi$ for an integer $k \in\{-n, \ldots, n\}$, then $\lambda$ is an eigenvalue, except if $k=0$, which corresponds to $\lambda=0$ and $p_{j+1 / 2}=q_{j+1 / 2}=0$ for all $j \in\{0, \ldots, n\}$. This gives us exactly $2 n$ eigenvalues $\pm \lambda^{k}, k \in\{1, \ldots, n\}$.

Moreover, the derivative of $f$ is explicit:

$$
f^{\prime}(\lambda)=8 \sum_{k=0}^{n} \frac{1}{4+\left(\lambda h_{k+1 / 2}\right)^{2}} h_{k+1 / 2} .
$$




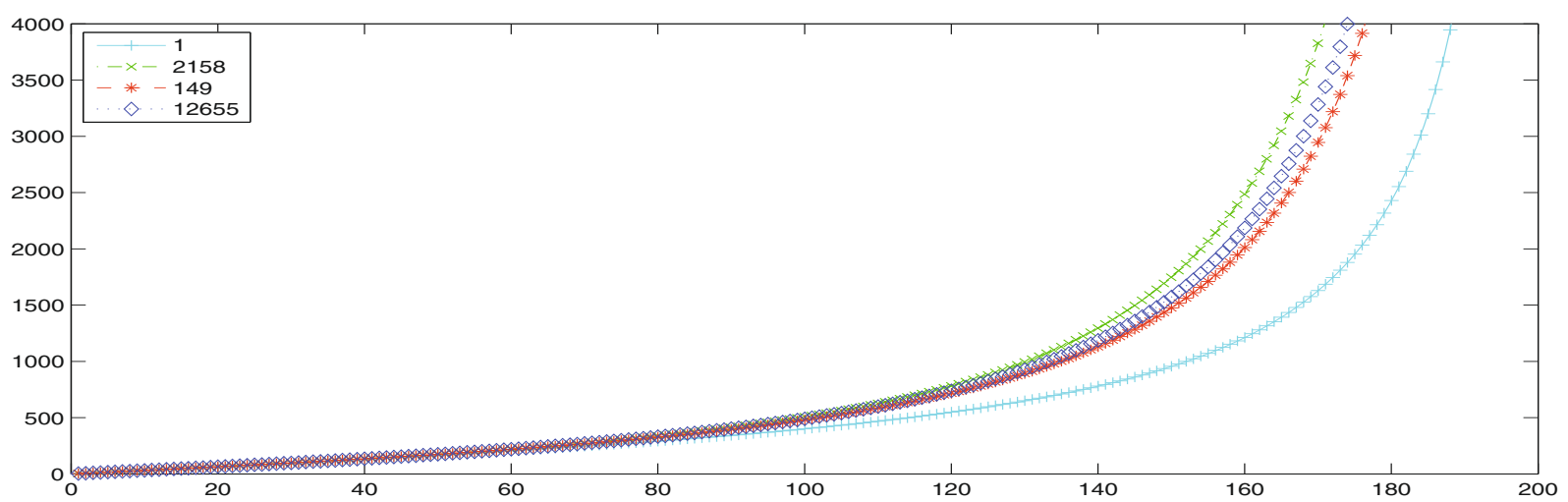

FIgURE 1. Dispersion diagrams for various meshes constituted by 200 points generated by Method 1 for different values of Reg.

It follows that

$$
0 \leq f^{\prime}(\lambda) \leq 2 \sum_{k=0}^{n} h_{k+1 / 2}=2
$$

Since all the eigenvalues are simple and $f\left(\lambda^{k+1}\right)-f\left(\lambda^{k}\right)=2 \pi$ for all $k \in\{1, \ldots, n-1\}$, this implies that the gap between the eigenvalues is bounded from below by $\pi$, and therefore (2.3) holds.

Using the concavity of arctan gives the following estimate:

$$
\arctan \left(\frac{\lambda^{k}}{2(n+1)}\right)=\arctan \left(\frac{1}{2(n+1)} \sum_{j=0}^{n} \lambda^{k} h_{j+1 / 2}\right) \geq \frac{1}{n+1} \sum_{j=0}^{n} \arctan \left(\frac{\lambda^{k} h_{j+1 / 2}}{2}\right)=\frac{k}{n+1} \frac{\pi}{2} .
$$

In other words,

$$
\lambda^{k} \geq 2(n+1) \tan \left(\frac{k}{n+1} \frac{\pi}{2}\right),
$$

and (2.4) follows. Indeed, the right hand-side inequality in (2.4) simply follows from the standard inequality $\tan (\eta) \geq \eta$ for $\eta \in[0, \pi / 2)$.

We illustrate this result in Figures 1 and 2 by computing dispersion diagrams for various nonuniform meshes $\mathcal{S}_{n}$, that we characterize by their regularity $\operatorname{Reg}\left(\mathcal{S}_{n}\right)$, as defined in $(1.9)$.

Let us briefly explain the two ways we have chosen for generating them.

- Method 1. In Figure 1, we create a random vector $h$ of length $n+1$ whose values are chosen according to a uniform law on $(0,1)$. This vector is then normalized such that the sum of its components is one, so that $h$ corresponds to the vector $\left(h_{1 / 2, n}, \ldots, h_{n+1 / 2, n}\right)$, which describes the mesh in a unique way.

- Method 2. In Figure 2, we create a random vector $x$ of length $n$ whose components are chosen according to a uniform law on $(0,1)$. Then we sort its components in an increasing way to obtain a vector $\left(x_{1, n}, \ldots, x_{n, n}\right)$, which represents the mesh points.

In both cases, the dispersion diagrams look the same. It is particularly striking that the shape of the dispersion diagrams does not seem to depend significantly on the meshes.

\subsection{Spectral properties on $M$-regular meshes}

This subsection is devoted to prove additional properties for the spectrum of (1.7) when the mesh $\mathcal{S}_{n}$ is $M$-regular for some $M \geq 1$. 


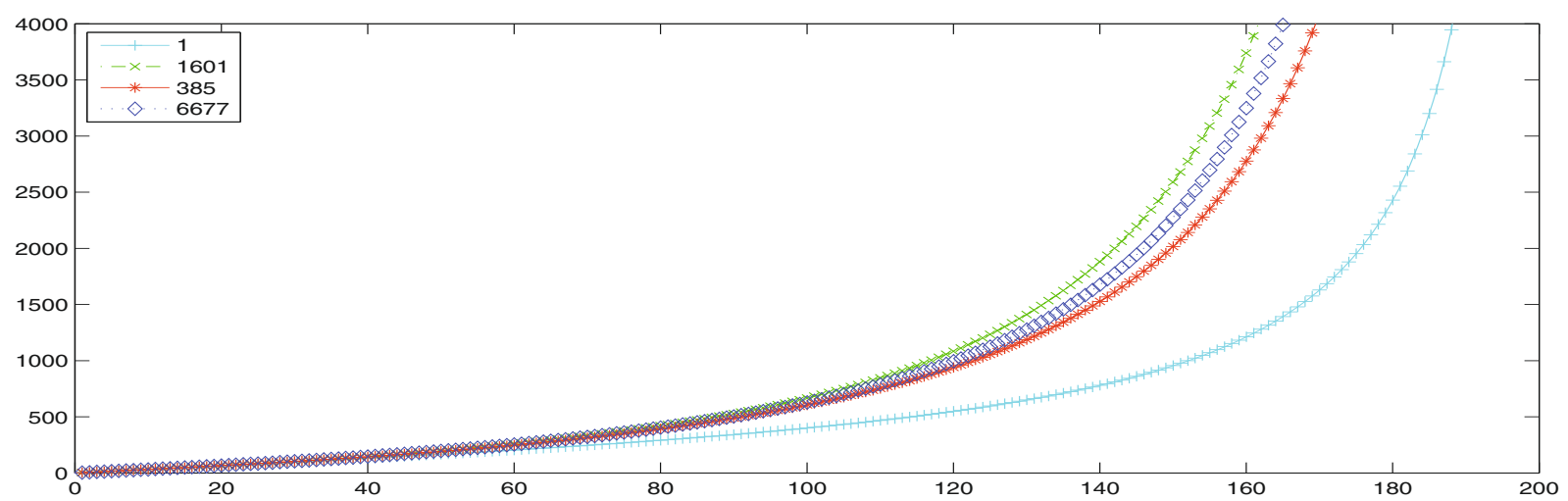

Figure 2. Dispersion diagrams for various meshes constituted by 200 points generated by Method 2 for different values of Reg.

Theorem 2.3. Let $M \geq 1$.

Then, for any $M$-regular mesh $\mathcal{S}_{n}$, the eigenvalue $\lambda_{n}^{n}$ of $(2.1)$ on $\mathcal{S}_{n}$ satisfies

$$
\lambda_{n}^{n} \leq \frac{4 M}{\pi}(n+1)^{2} .
$$

Besides, for any $M$-regular mesh $\mathcal{S}_{n}$, if $\phi_{n}^{k}$ denotes the eigenvector corresponding to $\lambda_{n}^{k}$ in (2.1), then its energy

$$
E_{n}^{k}=\frac{1}{2} \sum_{j=0}^{n} h_{j+1 / 2, n}\left(\left|\frac{\phi_{j+1, n}^{k}-\phi_{j, n}^{k}}{h_{j+1 / 2, n}}\right|^{2}+\left|\lambda_{n}^{k}\right|^{2}\left|\frac{\phi_{j, n}^{k}+\phi_{j+1, n}^{k}}{2}\right|^{2}\right)
$$

satisfies

$$
\frac{1}{1+M^{2}}\left(\left|\frac{\phi_{1, n}^{k}}{h_{1 / 2, n}}\right|^{2}+\frac{h_{1 / 2, n}^{2}}{4}\left|\frac{\lambda_{n}^{k} \phi_{1, n}^{k}}{h_{1 / 2, n}}\right|^{2}\right) \leq E_{n}^{k} \leq\left(1+M^{2}\right)\left(\left|\frac{\phi_{1, n}^{k}}{h_{1 / 2, n}}\right|^{2}+\frac{h_{1 / 2, n}^{2}}{4}\left|\frac{\lambda_{n}^{k} \phi_{1, n}^{k}}{h_{1 / 2, n}}\right|^{2}\right) .
$$

Moreover, if $\omega=(a, b)$ is some subinterval of $(0,1)$, then the energy of the $k$-th eigenvector $\phi_{n}^{k}$ in $\omega$, defined by

$$
E_{\omega, n}^{k}=\frac{1}{2} \sum_{x_{j, n} \in \omega} h_{j+1 / 2, n}\left(\left|\frac{\phi_{j+1, n}^{k}-\phi_{j, n}^{k}}{h_{j+1 / 2, n}}\right|^{2}+\left|\lambda_{n}^{k}\right|^{2}\left|\frac{\phi_{j, n}^{k}+\phi_{j+1, n}^{k}}{2}\right|^{2}\right)
$$

satisfies

$$
E_{n}^{k} \leq \frac{M^{2}}{|\omega|} E_{\omega, n}^{k} .
$$

Remark 2.4. These inequalities roughly say that the eigenvectors cannot concentrate in some part of an $M$-regular mesh. These properties are indeed the one needed for control and stabilization purposes, as we will see in next sections.

Remark 2.5. Note that Theorem 2.1 gives the estimate

$$
\lambda_{n}^{n} \geq 2(n+1) \tan \left(\left(1-\frac{1}{n+1}\right) \frac{\pi}{2}\right) \underset{n \rightarrow \infty}{\simeq} \frac{4}{\pi}(n+1)^{2} .
$$

Combined with estimate (2.11), this indicates that, when considering sequences of $M$-regular meshes, the eigenvalues $\lambda_{n}^{n}$ really grow as $n^{2}$ when $n \rightarrow \infty$. 
Proof. Along the proof, we fix an integer $n$, a real number $M \geq 1$ and an $M$-regular mesh $\mathcal{S}_{n}$, so that we can remove the index $n$ without confusion.

Inequality (2.11) is a consequence of (2.2). Indeed, if we set $h=\min \left\{h_{j+1 / 2}\right\}$ and $H=\max \left\{h_{j+1 / 2}\right\}$, then we have

$$
1 \leq(n+1) H \leq(n+1) M h .
$$

Besides, using (2.2), we get

$$
\sum_{j=0}^{n} \arctan \left(\frac{\lambda^{n} h_{j+1 / 2}}{2}\right)=\frac{n \pi}{2} \geq(n+1) \arctan \left(\frac{\lambda^{n} h}{2}\right)
$$

which provides

$$
\frac{\lambda^{n}}{(n+1)^{2}} \leq \frac{2}{h(n+1)^{2}} \tan \left(\frac{\pi}{2}\left(1-\frac{1}{n+1}\right)\right) \leq M \sup _{\eta \in[0,1]}\left\{2 \eta \tan \left(\frac{\pi}{2}(1-\eta)\right)\right\},
$$

from which (2.11) follows.

To derive the properties (2.13) and (2.15) of the eigenvectors, we use the computations and notations (2.5) introduced in the proof of Theorem 2.1. Namely, we introduce:

$$
p_{j+1 / 2}^{k}=\frac{\phi_{j+1}^{k}-\phi_{j}^{k}}{h_{j+1 / 2}}, \quad q_{j+1 / 2}^{k}=\frac{i \lambda^{k}}{2}\left(\phi_{j}^{k}+\phi_{j+1}^{k}\right), \quad j \in\{0, \ldots, n\} .
$$

Then the previous computations, and in particular identities (2.8)-(2.9), give:

$$
\begin{aligned}
E^{k} & =\frac{1}{2} \sum_{j=0}^{n} h_{j+1 / 2}\left(\left|p_{j+1 / 2}^{k}\right|^{2}+\left|q_{j+1 / 2}^{k}\right|^{2}\right) \\
& =\frac{1}{4} \sum_{j=0}^{n} h_{j+1 / 2}\left(\left|p_{j+1 / 2}^{k}-q_{j+1 / 2}^{k}\right|^{2}+\left|p_{j+1 / 2}^{k}+q_{j+1 / 2}^{k}\right|^{2}\right) \\
& =\frac{1}{4} \sum_{j=0}^{n} h_{j+1 / 2}\left(\left|\bar{\beta}_{j}\right|^{2}\left|p_{1 / 2}^{k}-q_{1 / 2}^{k}\right|^{2}+\left|\beta_{j}\right|^{2}\left|p_{1 / 2}^{k}+q_{1 / 2}^{k}\right|^{2}\right) \\
& =\frac{1}{4} \sum_{j=0}^{n} h_{j+1 / 2} \frac{4+\left(\lambda h_{1 / 2}\right)^{2}}{4+\left(\lambda h_{j+1 / 2}\right)^{2}}\left(\left|p_{1 / 2}^{k}-q_{1 / 2}^{k}\right|^{2}+\left|p_{1 / 2}^{k}+q_{1 / 2}^{k}\right|^{2}\right) .
\end{aligned}
$$

Using the definition $(2.5)$ of $\left(p_{1 / 2}^{k}, q_{1 / 2}^{k}\right)$, this leads to

$$
E^{k}=\frac{1}{2}\left(\sum_{j=0}^{n} \frac{h_{j+1 / 2}}{4+\left(\lambda^{k} h_{j+1 / 2}\right)^{2}}\right)\left(4+\left(\lambda^{k} h_{1 / 2}\right)^{2}\right)\left(\left|\frac{\phi_{1}^{k}}{h_{1 / 2}}\right|^{2}+\frac{h_{1 / 2}^{2}}{4}\left|\frac{\lambda^{k} \phi_{1}^{k}}{h_{1 / 2}}\right|^{2}\right) .
$$

Given an interval $\omega$, the same computations give for $E_{\omega}^{k}$ :

$$
E_{\omega}^{k}=\frac{1}{2}\left(\sum_{x_{j} \in \omega} \frac{h_{j+1 / 2}}{4+\left(\lambda^{k} h_{j+1 / 2}\right)^{2}}\right)\left(4+\left(\lambda^{k} h_{1 / 2}\right)^{2}\right)\left(\left|\frac{\phi_{1}^{k}}{h_{1 / 2}}\right|^{2}+\frac{h_{1 / 2}^{2}}{4}\left|\frac{\lambda^{k} \phi_{1}^{k}}{h_{1 / 2}}\right|^{2}\right) .
$$

Inequalities (2.13) and (2.15) easily follow from (2.17)-(2.18) and the $M$-regularity assumption. 


\subsection{Proof of Theorem $\mathbf{1 . 2}$}

Our strategy is based on Ingham's lemma on non-harmonic Fourier series, which we recall hereafter (see [18,30]):

Lemma 2.6 (Ingham's lemma). Let $\left(\lambda_{k}\right)_{k \in \mathbb{N}}$ be an increasing sequence of real numbers and $\gamma>0$ be such that

$$
\lambda_{k+1}-\lambda_{k} \geq \gamma>0, \forall k \in \mathbb{N}
$$

Then, for any $T>2 \pi / \gamma$, there exist two positive constants $c=c(T, \gamma)>0$ and $C=C(T, \gamma)>0$ such that, for any sequence $\left(a_{k}\right)_{k \in \mathbb{N}}$,

$$
c \sum_{k \in \mathbb{N}}\left|a_{k}\right|^{2} \leq \int_{0}^{T}\left|\sum_{k \in \mathbb{N}} a_{k} \mathrm{e}^{\mathrm{i} \lambda_{k} t}\right|^{2} \mathrm{~d} t \leq C \sum_{k \in \mathbb{N}}\left|a_{k}\right|^{2}
$$

Proof of Theorem 1.2. Let us consider a sequence $\left(\mathcal{S}_{n}\right)_{n}$ of $M$-regular meshes.

According to inequality (2.3), the gap condition (2.19) holds with $\gamma=\pi$. Thus, due to Lemma 2.6, we only need to prove the observability inequalities (1.11)-(1.12) for the stationary solutions

$$
u_{n}^{k}(t)=\exp \left(\mathrm{i} \lambda_{n}^{k} t\right) \phi_{n}^{k}
$$

of (1.7) corresponding to the eigenvectors $\phi_{n}^{k}$ of system $(2.1)$ on $\mathcal{S}_{n}$.

Since each mesh $\mathcal{S}_{n}$ is $M$-regular, we can apply Theorem 2.3. Especially, inequality (2.13) holds, and therefore Ingham's inequality (2.20) directly implies (1.11).

To prove (1.12), we fix $J=(a, b) \subset(0,1)$ a subinterval of $(0,1)$. According to Ingham's lemma and (2.3), it is sufficient to prove that there exists a constant $C$ independent of $n$ and $k$ such that, for any eigenvector $\phi_{n}^{k}$ solution of (2.1) on $\mathcal{S}_{n}$ corresponding to the eigenvalue $\lambda_{n}^{k}$, the quantity

$$
I_{J, n}^{k}=\sum_{x_{j, n} \in J} h_{j+1 / 2, n}\left|\lambda_{n}^{k}\right|^{2}\left(\frac{\phi_{j, n}^{k}+\phi_{j+1, n}^{k}}{2}\right)^{2}
$$

satisfies

$$
E_{n}^{k} \leq C I_{J, n}^{k}
$$

We thus investigate inequality $(2.22)$ on a mesh $\mathcal{S}_{n}$ by using a multiplier technique.

Let $\omega$ be a strict subinterval of $J$ and let us denote by $\eta$ a function of $x \in[0,1]$ such that:

$$
\left\{\begin{array} { l } 
{ \eta ( x ) = 0 , \quad \forall x \in ( 0 , 1 ) \backslash J , } \\
{ \eta ( x ) = 1 , \quad \forall x \in \omega , }
\end{array} \quad \left\{\begin{array}{l}
\|\eta\|_{\infty} \leq 1 \\
\left\|\eta^{\prime}\right\|_{\infty} \leq C_{J, \omega}
\end{array}\right.\right.
$$

To simplify notation, we drop the exponent $k$ and the index $n$ hereafter. Below, we denote by $\eta_{j}$ the value of $\eta$ in the mesh point $x_{j}$.

We consider system (2.1) and multiply each equation by $\eta_{j}^{2} \phi_{j}$. Discrete integrations by parts yield:

$$
\lambda^{2} \sum_{j=0}^{n} h_{j+1 / 2}\left(\frac{\phi_{j}+\phi_{j+1}}{2}\right)\left(\frac{\eta_{j}^{2} \phi_{j}+\eta_{j+1}^{2} \phi_{j+1}}{2}\right)=\sum_{j=0}^{n} h_{j+1 / 2}\left(\frac{\phi_{j+1}-\phi_{j}}{h_{j+1 / 2}}\right)\left(\frac{\eta_{j+1}^{2} \phi_{j+1}-\eta_{j}^{2} \phi_{j}}{h_{j+1 / 2}}\right)
$$

Then we deduce that

$$
\lambda^{2} \sum_{j=0}^{n} h_{j+1 / 2}\left(\frac{\eta_{j}^{2}+\eta_{j+1}^{2}}{2}\right)\left(\frac{\phi_{j}+\phi_{j+1}}{2}\right)^{2}-\sum_{j=0}^{n} h_{j+1 / 2}\left(\frac{\eta_{j}^{2}+\eta_{j+1}^{2}}{2}\right)\left(\frac{\phi_{j+1}-\phi_{j}}{h_{j+1 / 2}}\right)^{2}=A_{1}+A_{2}
$$


where $A_{1}$ and $A_{2}$ are defined by

$$
\begin{aligned}
& A_{1}=-\frac{\lambda^{2}}{2} \sum_{j=0}^{n} h_{j+1 / 2}^{3}\left(\frac{\phi_{j}+\phi_{j+1}}{2}\right)\left(\frac{\phi_{j+1}-\phi_{j}}{h_{j+1 / 2}}\right)\left(\frac{\eta_{j+1}-\eta_{j}}{h_{j+1 / 2}}\right)\left(\frac{\eta_{j}+\eta_{j+1}}{2}\right), \\
& A_{2}=2 \sum_{j=0}^{n} h_{j+1 / 2}\left(\frac{\phi_{j}+\phi_{j+1}}{2}\right)\left(\frac{\phi_{j+1}-\phi_{j}}{h_{j+1 / 2}}\right)\left(\frac{\eta_{j+1}-\eta_{j}}{h_{j+1 / 2}}\right)\left(\frac{\eta_{j}+\eta_{j+1}}{2}\right) .
\end{aligned}
$$

Then, for any choices of positive parameters $\delta_{1}$ and $\delta_{2}$, we get:

$$
\begin{aligned}
& \left|A_{1}\right| \leq \frac{1}{4 \delta_{1}} \sum_{j=0}^{n} h_{j+1 / 2} \lambda^{2}\left(\frac{\phi_{j}+\phi_{j+1}}{2}\right)^{2}\left(\frac{\eta_{j+1}-\eta_{j}}{h_{j+1 / 2}}\right)^{2}+\frac{\delta_{1}}{4} \sum_{j=0}^{n} h_{j+1 / 2}\left(\lambda^{2} h_{j+1 / 2}^{4}\right)\left(\frac{\phi_{j+1}-\phi_{j}}{h_{j+1 / 2}}\right)^{2}\left(\frac{\eta_{j}+\eta_{j+1}}{2}\right)^{2}, \\
& \left|A_{2}\right| \leq \frac{1}{\delta_{2}} \sum_{j=0}^{n} h_{j+1 / 2}\left(\frac{\phi_{j}+\phi_{j+1}}{2}\right)^{2}\left(\frac{\eta_{j+1}-\eta_{j}}{h_{j+1 / 2}}\right)^{2}+\delta_{2} \sum_{j=0}^{n} h_{j+1 / 2}\left(\frac{\phi_{j+1}-\phi_{j}}{h_{j+1 / 2}}\right)^{2}\left(\frac{\eta_{j}+\eta_{j+1}}{2}\right)^{2} .
\end{aligned}
$$

Using that

$$
\left(\frac{n+1}{M}\right) \sup h_{j+1 / 2} \leq(n+1) \inf h_{j+1 / 2} \leq 1
$$

estimate (2.11) gives

Therefore, if we set

$$
\lambda^{2} h_{j+1 / 2}^{4} \leq\left(\frac{4 M}{\pi}(n+1)^{2}\right)^{2}\left(\frac{M}{(n+1)}\right)^{4} \leq\left(\frac{4}{\pi}\right)^{2} M^{4} .
$$

using the classical inequality

$$
\delta_{1}=\frac{\pi^{2}}{16 M^{4}} ; \quad \delta_{2}=\frac{1}{4}
$$

$$
\left(\frac{\eta_{j}+\eta_{j+1}}{2}\right)^{2} \leq \frac{\eta_{j}^{2}+\eta_{j+1}^{2}}{2}
$$

we deduce from (2.24) the existence of two constants independent of $k$ and $n$ such that

$$
\begin{aligned}
\frac{1}{2} \sum_{j=0}^{n} h_{j+1 / 2}\left(\frac{\eta_{j}^{2}+\eta_{j+1}^{2}}{2}\right)\left(\frac{\phi_{j+1}-\phi_{j}}{h_{j+1 / 2}}\right)^{2} \leq \lambda^{2} \sum_{j=0}^{n} h_{j+1 / 2}\left(\frac{\eta_{j}^{2}+\eta_{j+1}^{2}}{2}\right)\left(\frac{\phi_{j}+\phi_{j+1}}{2}\right)^{2} \\
+C_{1} \sum_{j=0}^{n} h_{j+1 / 2} \lambda^{2}\left(\frac{\phi_{j}+\phi_{j+1}}{2}\right)^{2}\left(\frac{\eta_{j+1}-\eta_{j}}{h_{j+1 / 2}}\right)^{2}+C_{2} \sum_{j=0}^{n} h_{j+1 / 2}\left(\frac{\phi_{j}+\phi_{j+1}}{2}\right)^{2}\left(\frac{\eta_{j+1}-\eta_{j}}{h_{j+1 / 2}}\right)^{2} .
\end{aligned}
$$

But $|\lambda|$ is also uniformly bounded from below (see (2.4)), and therefore we obtain that

$$
\begin{aligned}
\sum_{j=0}^{n} h_{j+1 / 2}\left(\frac{\eta_{j}^{2}+\eta_{j+1}^{2}}{2}\right)\left(\frac{\phi_{j+1}-\phi_{j}}{h_{j+1 / 2}}\right)^{2} \leq \lambda^{2} \sum_{j=0}^{n} h_{j+1 / 2}\left(\frac{\eta_{j}^{2}+\eta_{j+1}^{2}}{2}\right)\left(\frac{\phi_{j}+\phi_{j+1}}{2}\right)^{2} & \\
& +C \sum_{j=0}^{n} h_{j+1 / 2} \lambda^{2}\left(\frac{\phi_{j}+\phi_{j+1}}{2}\right)^{2}\left(\frac{\eta_{j+1}-\eta_{j}}{h_{j+1 / 2}}\right)^{2} .
\end{aligned}
$$

Using the properties (2.23) of the function $\eta$ leads us to the following result:

$$
E_{\omega, n}^{k} \leq C I_{J, n}^{k} .
$$

Therefore inequality (2.22) can be deduced from inequality (2.15) applied to $\omega$. 


\subsection{The regularity assumption}

Let us discuss the assumption on the regularity of the meshes.

\subsubsection{Concentration effects without the $M$-regularity assumption}

Here, we design a sequence of meshes $\mathcal{S}_{n}$ such that:

- The sequence $\operatorname{Reg}\left(\mathcal{S}_{n}\right)$ goes to infinity arbitrarily slowly when $n \rightarrow \infty$.

- There exists an interval $J=[a, b]$ for which there is no constant $C$ such that for all $n$, for all eigenvectors $\phi_{n}^{k}$ of $(2.1)$ on $\mathcal{S}_{n}$,

$$
E_{n}^{k} \leq C E_{J, n}^{k}
$$

where $E_{n}^{k}$ and $E_{J, n}^{k}$ are, respectively, as in (2.12) and (2.14).

Note that (2.25) constitutes an obstruction for (1.12) to hold.

Choose a strict non-empty closed subinterval $J$ of $(0,1)$, and a sequence $K_{n}$ going to infinity when $n \rightarrow \infty$. Introduce a sequence of meshes $\left(\mathcal{S}_{n}\right)$, each one constituted by $n+2$ points such that

$$
x_{0, n}=0, \quad x_{n+1, n}=1, \quad \begin{cases}x_{j+1, n}-x_{j, n}=H_{n}, & \text { if }\left[x_{j, n}, x_{j+1, n}\right] \subset J, \\ x_{j+1, n}-x_{j, n}=h_{n}, & \text { if }\left[x_{j, n}, x_{j+1, n}\right] \subset[0,1] \backslash J,\end{cases}
$$

where $H_{n}=K_{n} h_{n}$. Remark that the mesh $\mathcal{S}_{n}$ is then totally described by the quantity $K_{n}$. From identities (2.17)-(2.18), we get:

But

$$
\frac{E_{n}^{k}}{E_{J, n}^{k}}=1+\frac{E_{(0,1) \backslash J, n}^{k}}{E_{J, n}^{k}}=1+\frac{1-|J|}{|J|} \frac{4+\left(\lambda_{n}^{k} H_{n}\right)^{2}}{4+\left(\lambda_{n}^{k} h_{n}\right)^{2}}
$$

$$
\frac{|J|}{H_{n}}+\frac{1-|J|}{h_{n}}=n+1
$$

and so $(n+1) h_{n}=(1-|J|)+|J| / K_{n}$ converges to $1-|J|$ when $n \rightarrow \infty$. But inequality (2.4) gives

$$
\frac{\lambda_{n}^{n} h_{n}}{2} \geq(n+1) h_{n} \tan \left(\frac{n}{n+1} \frac{\pi}{2}\right)
$$

and then $\left(\lambda_{n}^{n} h_{n}\right)_{n}$ goes to infinity when $n \rightarrow \infty$. Especially, this implies that

$$
\frac{E_{n}^{n}}{E_{J, n}^{n}} \underset{n \rightarrow \infty}{\simeq} \frac{1-|J|}{|J|} \frac{H_{n}^{2}}{h_{n}^{2}}=\frac{1-|J|}{|J|} K_{n}^{2} \rightarrow \infty
$$

and therefore there is no constant such that (2.25) holds uniformly with respect to $n \in \mathbb{N}$ and $k \in\{1, \ldots, n\}$.

\subsubsection{Partial regularity assumptions}

Without the $M$-regularity assumption, one can derive partial results, due to the explicit form (2.17) of the energy.

For instance, identity (2.17) on the energy of the $k$-th eigenvector $\phi_{n}^{k}$ on $\mathcal{S}_{n}$ gives:

$$
E_{n}^{k} \leq \frac{4+\left(\lambda_{n}^{k} h_{1 / 2, n}\right)^{2}}{4+\inf _{j}\left(\lambda_{n}^{k} h_{j+1 / 2, n}\right)^{2}}\left(\left|\frac{\phi_{1, n}^{k}}{h_{1 / 2, n}}\right|^{2}+\frac{h_{1 / 2, n}^{2}}{4}\left|\frac{\lambda_{n}^{k} \phi_{1, n}^{k}}{h_{1 / 2, n}}\right|^{2}\right) .
$$

In particular, if there exists a constant $M_{1}>0$ such that for all $n$,

$$
h_{1 / 2, n} \leq M_{1} \inf _{j} h_{j+1 / 2, n},
$$


then for all $n$ and $k$,

$$
E_{n}^{k} \leq\left(1+M_{1}^{2}\right)\left(\left|\frac{\phi_{1, n}^{k}}{h_{1 / 2, n}}\right|^{2}+\frac{h_{1 / 2, n}^{2}}{4}\left|\frac{\lambda_{n}^{k} \phi_{1, n}^{k}}{h_{1 / 2, n}}\right|^{2}\right) .
$$

Now, consider the reverse equality. From (2.17), we get

$$
E_{n}^{k} \geq \frac{4+\left(\lambda_{n}^{k} h_{1 / 2, n}\right)^{2}}{4+\sup _{j}\left(\lambda_{n}^{k} h_{j+1 / 2, n}\right)^{2}}\left(\left|\frac{\phi_{1, n}^{k}}{h_{1 / 2, n}}\right|^{2}+\frac{h_{1 / 2, n}^{2}}{4}\left|\frac{\lambda_{n}^{k} \phi_{1, n}^{k}}{h_{1 / 2, n}}\right|^{2}\right) .
$$

In particular, if there exists a constant $M_{2}>0$ such that for all $n$,

$$
\sup _{j} h_{j+1 / 2, n} \leq M_{2} h_{1 / 2, n}
$$

then, for all $n$ and $k$, we get

$$
E_{n}^{k} \geq \frac{1}{1+M_{2}^{2}}\left(\left|\frac{\phi_{1, n}^{k}}{h_{1 / 2, n}}\right|^{2}+\frac{h_{1 / 2, n}^{2}}{4}\left|\frac{\lambda_{n}^{k} \phi_{1, n}^{k}}{h_{1 / 2, n}}\right|^{2}\right)
$$

Besides, as in Section 2.4.1, for each integer $n$, we can consider sequences of meshes $\mathcal{S}_{n}$ given as in (1.6) defined by

$$
x_{1, n}-x_{0, n}=h_{1 / 2, n}, \quad x_{j+1, n}-x_{j, n}=h_{n}, \quad \forall j \in\{1, \ldots, n\},
$$

where $h_{1 / 2, n}$ and $h_{n}$ are two sequences going to zero. It is then easy to check that if condition (2.27) is not satisfied, that is if $h_{n} / h_{1 / 2, n} \rightarrow \infty$ when $n \rightarrow \infty$, then there is no positive constant $c$ such that

$$
E_{n}^{k} \geq c\left(\left|\frac{\phi_{1, n}^{k}}{h_{1 / 2, n}}\right|^{2}+\frac{h_{1 / 2, n}^{2}}{4}\left|\frac{\lambda_{n}^{k} \phi_{1, n}^{k}}{h_{1 / 2, n}}\right|^{2}\right)
$$

uniformly in $k$ and $n$.

On the contrary, if $h_{n} / h_{1 / 2, n} \rightarrow 0$ when $n \rightarrow \infty$, then there is no constant $C$ such that

$$
E_{n}^{k} \leq C\left(\left|\frac{\phi_{1, n}^{k}}{h_{1 / 2, n}}\right|^{2}+\frac{h_{1 / 2, n}^{2}}{4}\left|\frac{\lambda_{n}^{k} \phi_{1, n}^{k}}{h_{1 / 2, n}}\right|^{2}\right)
$$

uniformly in $k$ and $n$.

Therefore, if we consider a sequence of meshes $\mathcal{S}_{n}$ such that $\operatorname{Reg}\left(\mathcal{S}_{n}\right)$ is unbounded, we cannot expect in general to have both observability and admissibility properties (1.11) uniformly with respect to $n$.

Remark 2.7. If we are interested in the observability inequality (1.12) for a particular subinterval $(a, b) \subset(0,1)$, the situation is more intricate. As above, due to the explicit description of the energies (2.17) and (2.18), one easily checks that if there exists a constant $M_{3}$ such that for all $n \in \mathbb{N}$,

$$
\sup _{x_{j, n} \in(a, b)}\left\{h_{j+1 / 2, n}\right\} \leq M_{3} \inf _{x_{j, n} \notin(a, b)}\left\{h_{j+1 / 2, n}\right\},
$$

then for all $n \in \mathbb{N}$ and for all $k \in\{1, \ldots, n\}$,

$$
E_{n}^{k} \leq\left(1+\frac{M_{3}^{2}}{(b-a)}\right) E_{(a, b), n}^{k}
$$



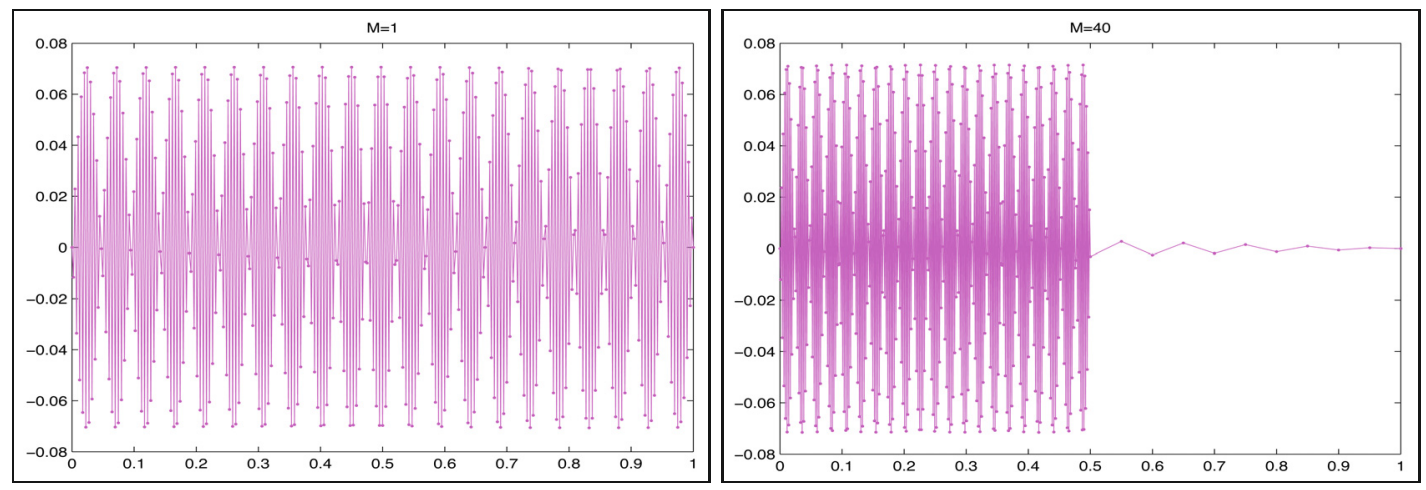

Figure 3. Concentration effects of the 380-th eigenvector on the mesh constructed in Section 2.4.1 with $n=400$ and $J=(1 / 2,1)$ : left $M=1$ (uniform mesh), and right $M=40$.

However, under the only condition (2.28), the estimates (2.11) on the eigenvalues might be false, and therefore the proof presented above of inequality (2.22) (with $J=(a, b)$ ) fails. We do not know if assumption (2.28) suffices to guarantee (2.22) to hold uniformly with respect to $n \in \mathbb{N}$ and $k \in\{1, \ldots, n\}$.

Also remark that if assumption (2.28) holds for a sequence of meshes $\mathcal{S}_{n}$ for any subinterval $(a, b) \subset(0,1)$, then there exists a real number $M$ such that all the meshes $\mathcal{S}_{n}$ are $M$-regular.

Remark 2.8. We emphasize that, for the various meshes constructed in this subsection to show the optimality of our results, the corresponding eigenvalues do satisfy the gap condition (2.3), as proved in Theorem 2.1 . Actually, the corresponding dispersion diagrams are very close to the ones presented in Figures 1 and 2 . In particular, uniform admissibility and observability properties do fail only because of concentration effects for the eigenvectors.

To illustrate this fact, we present in Figure 3 the behavior of the 380-th eigenvector of a mesh obtained for $n=400$, constructed such that:

- $x_{j+1, n}-x_{j, n}=h_{n}$ for $\left[x_{j, n}, x_{j+1, n}\right] \subset[0,1 / 2]$;

- $x_{j+1, n}-x_{j, n}=H_{n}$ for $\left[x_{j, n}, x_{j+1, n}\right] \subset[1 / 2,1]$;

- $H_{n}=M h_{n}$.

In Figure 3, we compare the eigenvector obtained for $M=1$, which corresponds to the one on a uniform mesh, and the one obtained for $M=40$, for which, as expected by the computations led in Section 2.4.1, we observe a concentration phenomenon in the part where the mesh size is smaller.

\section{Application to the null Controllability of the Wave equation}

\subsection{The continuous setting}

Let us first present the problem. It is well-known that for any time $T \geq 2$, given any initial data $\left(y^{0}, y^{1}\right) \in$ $L^{2}(0,1) \times H^{-1}(0,1)$, we can find a control function $v(t) \in L^{2}(0, T)$ such that the solution of

$$
\begin{cases}\partial_{t t}^{2} y-\partial_{x x}^{2} y=0, & (x, t) \in(0,1) \times(0, T), \\ y(0, t)=v(t), y(1, t)=0, & t \in(0, T), \\ y(x, 0)=y^{0}(x), \partial_{t} y(x, 0)=y^{1}(x), & x \in(0,1)\end{cases}
$$

satisfies

$$
y(T)=0, \quad \partial_{t} y(T)=0 .
$$

By duality (namely the Hilbert Uniqueness Method, or HUM in short), this property is equivalent to the observability inequality (1.4), see [21]. 
Note that there might be several controls $v \in L^{2}(0, T)$ such that (3.2) holds for solutions of (3.1). In the sequel, we will say that such a $v$ is an admissible control for (3.1).

Besides, there is an explicit method to compute the so-called HUM control $v_{H U M}$, which is the one of minimal $L^{2}(0, T)$-norm among all admissible controls for (3.1). Indeed, set $T \geq 2$ and consider the functional

$$
\begin{aligned}
& \mathcal{J}: H_{0}^{1}(0,1) \times L^{2}(0,1) \rightarrow \mathbb{R} \\
& \mathcal{J}\left(z^{0}, z^{1}\right)=\frac{1}{2} \int_{0}^{T}\left(\partial_{x} z\right)^{2}(0, t) \mathrm{d} t-\int_{0}^{1} y^{0}(x) \partial_{t} z(x, 0) \mathrm{d} x+\left\langle y^{1}, z(., 0)\right\rangle_{H^{-1} \times H_{0}^{1}},
\end{aligned}
$$

where $z$ is the solution of the backward conservative wave equation

$$
\begin{cases}\partial_{t t}^{2} z-\partial_{x x}^{2} z=0, & (x, t) \in(0,1) \times(0, T) \\ z(0, t)=z(1, t)=0, & t \in(0, T) \\ z(x, T)=z^{0}(x), \partial_{t} z(x, T)=z^{1}(x), & x \in(0,1)\end{cases}
$$

Then $\mathcal{J}$ is strictly convex, coercive (see (1.4)), and therefore has a unique minimizer $\left(Z^{0}, Z^{1}\right) \in H_{0}^{1}(0,1) \times$ $L^{2}(0,1)$. The HUM control is then given by $v_{H U M}(t)=\partial_{x} Z(0, t)$, where $Z$ is the solution of (3.4) with initial $\operatorname{data}\left(Z^{0}, Z^{1}\right)$.

Note also that the HUM control is the only admissible control $v$ for (3.1) that can be written as $v(t)=\partial_{x} z(0, t)$ for some $z$ solution of (3.4) with initial data in $H_{0}^{1}(0,1) \times L^{2}(0,1)$.

It is then natural to try to compute this control numerically. This question will be investigated in the sequel.

\subsection{The semi-discrete setting}

This part is inspired in $[5,6]$ where similar results have been derived for uniform meshes.

We consider a mesh $\mathcal{S}_{n}$ as in (1.6) and derive an approximation scheme for (3.1) from a mixed finite element method. The problem reads as follows: Given $y_{n}^{0}$ and $y_{n}^{1}$ defined on $\mathcal{S}_{n}$, find a discrete control $v_{n} \in L^{2}(0, T)$ such that the solution $y_{n}$ of

$$
\left\{\begin{array}{l}
\frac{h_{j-1 / 2, n}}{4}\left(y_{j-1, n}^{\prime \prime}+y_{j, n}^{\prime \prime}\right)+\frac{h_{j+1 / 2, n}}{4}\left(y_{j, n}^{\prime \prime}+y_{j+1, n}^{\prime \prime}\right) \\
=\frac{y_{j+1, n}-y_{j, n}}{h_{j+1 / 2, n}}-\frac{y_{j, n}-y_{j-1, n}}{h_{j-1 / 2, n}}, \quad j=1, \ldots, n, t \in[0, T] \\
y_{0, n}(t)=v_{n}(t), \quad y_{n+1, n}(t)=0, \quad t \in(0, T) \\
y_{j, n}(0)=y_{j, n}^{0} \quad y_{j, n}^{\prime}(0)=y_{j, n}^{1}, \quad j=1, \ldots, n
\end{array}\right.
$$

satisfies

$$
y_{j, n}(T)=0, \quad y_{j, n}^{\prime}(T)=0, \quad j=1, \ldots, n
$$

Again, the study of this problem is based on a duality principle. Given any $T>2$, we choose $\epsilon>0$ such that $T-4 \epsilon>2$ and a smooth function $\rho$ satisfying

$$
\left\{\begin{array}{ll}
\rho(t)=1, & \text { if } t \in[2 \epsilon, T-2 \epsilon] \\
\rho(t)=0, & \text { if } t \in[0, \epsilon] \cup[T-\epsilon, T],
\end{array} \quad \text { and } \quad 0 \leq \rho(t) \leq 1, \quad \forall t\right.
$$


We then introduce the functional $\mathcal{J}_{n}$ defined by:

$$
\begin{aligned}
\mathcal{J}_{n}\left(z_{n}^{0}, z_{n}^{1}\right)=\frac{1}{8} \int_{0}^{T} \rho(t)\left|z_{1, n}^{\prime}\right|^{2}( & t) \mathrm{d} t+\frac{1}{2} \int_{0}^{T}\left(\frac{z_{1, n}(t)}{h_{1 / 2, n}}\right)^{2} \mathrm{~d} t \\
& +\left(\frac{h_{1 / 2, n}}{4} y_{1, n}^{1} z_{1, n}(0)+\sum_{j=1}^{n} \frac{h_{j+1 / 2, n}}{4}\left(y_{j, n}^{1}+y_{j+1, n}^{1}\right)\left(z_{j, n}(0)+z_{j+1, n}(0)\right)\right) \\
& -\left(\frac{h_{1 / 2, n}}{4} y_{1, n}^{0} z_{1, n}^{\prime}(0)+\sum_{j=1}^{n} \frac{h_{j+1 / 2, n}}{4}\left(y_{j, n}^{0}+y_{j+1, n}^{0}\right)\left(z_{j, n}^{\prime}(0)+z_{j+1, n}^{\prime}(0)\right)\right),
\end{aligned}
$$

where $z_{n}$ is the solution of

$$
\left\{\begin{array}{l}
\frac{h_{j-1 / 2, n}}{4}\left(z_{j-1, n}^{\prime \prime}+z_{j, n}^{\prime \prime}\right)+\frac{h_{j+1 / 2, n}}{4}\left(z_{j, n}^{\prime \prime}+z_{j+1, n}^{\prime \prime}\right) \\
=\frac{z_{j+1, n}-z_{j, n}}{h_{j+1 / 2, n}}-\frac{z_{j, n}-z_{j-1, n}}{h_{j-1 / 2, n}}, \quad j=1, \ldots, n, t \in[0, T], \\
z_{0, n}(t)=z_{n+1, n}(t)=0, \quad t \in(0, T), \\
z_{j, n}(T)=z_{j, n}^{0}, \quad z_{j, n}^{\prime}(T)=z_{j, n}^{1}, \quad j=1, \ldots, n .
\end{array}\right.
$$

Then the following lemma holds:

Lemma 3.1. For any integer $n$, the functional $\mathcal{J}_{n}$ is strictly convex and coercive, and then has a unique minimizer $\left(Z_{n}^{0}, Z_{n}^{1}\right)$. Besides, for all $n$, if $v_{n}$ is the solution of

$$
\left\{\begin{array}{l}
-\frac{h_{1 / 2, n}}{4} v_{n}^{\prime \prime}+\frac{1}{h_{1 / 2, n}} v_{n}=-\frac{1}{4}\left(\rho Z_{1, n}^{\prime}\right)^{\prime}+\frac{1}{h_{1 / 2, n}^{2}} Z_{1, n}, \quad t \in[0, T] \\
v_{n}^{\prime}(0)=v_{n}^{\prime}(T)=0
\end{array}\right.
$$

where $Z_{n}$ is the solution of (3.9) with initial data $\left(Z_{n}^{0}, Z_{n}^{1}\right)$, then $v_{n}(t)$ is a control of (3.5) in time $T$.

The proof of Lemma 3.1 is the same as in [5]. For completeness, we will give a sketch of the proof hereafter.

For convenience, we introduce the operators $\mathbb{P}_{\mathcal{S}_{n}}, \mathbb{Q}_{\mathcal{S}_{n}}$ and $\mathbb{R}_{\mathcal{S}_{n}}$ which map discrete data $a_{n}=\left(a_{j, n}\right)_{j \in\{1, \ldots, n\}}$ given on a mesh $\mathcal{S}_{n}$ as in (1.6) to functions defined on $(0,1)$ by:

$$
\begin{aligned}
& \mathbb{P}_{\mathcal{S}_{n}} a_{n}(x)=a_{j, n}+\left(a_{j+1, n}-a_{j, n}\right)\left(\frac{x-x_{j, n}}{h_{j+1 / 2, n}}\right), \\
& \mathbb{Q}_{\mathcal{S}_{n}} a_{n}(x)=\frac{a_{j, n}+a_{j+1, n}}{2}, \\
& \mathbb{R}_{\mathcal{S}_{n}} a_{n}(x)=\frac{h_{j+1 / 2, n}}{4}\left(a_{j, n}+a_{j+1, n}\right)+\sum_{k=j+1}^{n} h_{k+1 / 2, n}\left(\frac{a_{k, n}+a_{k+1, n}}{2}\right),
\end{aligned} \text { on }\left[x_{j, n}, x_{j+1, n}\right],
$$

with the convention $a_{0, n}=a_{n+1, n}=0$. With these definitions, $\mathbb{P}_{\mathcal{S}_{n}}$ and $\mathbb{Q}_{\mathcal{S}_{n}}$ are extension operators, and $\mathbb{R}_{\mathcal{S}_{n}}$ corresponds to a piecewise continuous approximation operator of the discrete integrals $x \mapsto \int_{x}^{1} \mathbb{Q}_{\mathcal{S}_{n}} a_{n}(s) \mathrm{d} s$.

Let us rewrite all discrete computations in terms of the operators $\mathbb{P}_{\mathcal{S}_{n}}, \mathbb{Q}_{\mathcal{S}_{n}}, \mathbb{R}_{\mathcal{S}_{n}}$. First, for any solution $z_{n}$ of (3.9), the energy (1.8) writes

$$
E_{n}(t)=\frac{1}{2}\left\|\mathbb{Q}_{\mathcal{S}_{n}} z_{n}(t)\right\|_{L^{2}(0,1)}^{2}+\frac{1}{2}\left\|\partial_{x}\left(\mathbb{P}_{\mathcal{S}_{n}} z_{n}(t)\right)\right\|_{L^{2}(0,1)}^{2}
$$


Second, the functional $\mathcal{J}_{n}$ reads as

$$
\begin{aligned}
\mathcal{J}_{n}\left(z_{n}^{0}, z_{n}^{1}\right)=\frac{1}{8} \int_{0}^{T} \rho(t)\left|z_{1, n}^{\prime}\right|^{2}(t) \mathrm{d} t+\frac{1}{2} \int_{0}^{T} & \left(\frac{z_{1, n}(t)}{h_{1 / 2, n}}\right)^{2} \mathrm{~d} t \\
& +\int_{0}^{1}\left(\mathbb{R}_{\mathcal{S}_{n}} y_{n}^{1}\right)\left(\partial_{x} \mathbb{P}_{\mathcal{S}_{n}} z_{n}(0)\right) \mathrm{d} x-\int_{0}^{1}\left(\mathbb{Q}_{\mathcal{S}_{n}} y_{n}^{0}\right)\left(\mathbb{Q}_{\mathcal{S}_{n}} z_{n}^{\prime}(0)\right) \mathrm{d} x
\end{aligned}
$$

We are now in position to sketch the proof of Lemma 3.1.

Sketch of the proof of Lemma 3.1. Fix an integer $n \in \mathbb{N}$. The functional $\mathcal{J}_{n}$ is strictly convex, and its coercivity is obvious since we are working in a finite dimensional setting. It follows that $\mathcal{J}_{n}$ has a unique minimizer $\left(Z_{n}^{0}, Z_{n}^{1}\right)$.

Let us compute the Fréchet derivative of $\mathcal{J}_{n}$ in the minimizer $\left(Z_{n}^{0}, Z_{n}^{1}\right)$ : For any $\left(z_{n}^{0}, z_{n}^{1}\right)$, the solution $z_{n}$ of (3.9) on $\mathcal{S}_{n}$ satisfies (recall the definition (3.7) of $\rho$ ):

$$
\begin{aligned}
0=\int_{0}^{T}\left(-\frac{1}{4}\left(\rho(t) Z_{1, n}^{\prime}(t)\right)^{\prime}\right. & \left.+\frac{1}{h_{1 / 2, n}^{2}} Z_{1, n}(t)\right) z_{1, n}(t) \mathrm{d} t \\
& \quad+\int_{0}^{1}\left(\mathbb{R}_{\mathcal{S}_{n}} y_{n}^{1}\right)\left(\partial_{x} \mathbb{P}_{\mathcal{S}_{n}} z_{n}(0)\right) \mathrm{d} x-\int_{0}^{1}\left(\mathbb{Q}_{\mathcal{S}_{n}} y_{n}^{0}\right)\left(\mathbb{Q}_{\mathcal{S}_{n}} z_{n}^{\prime}(0)\right) \mathrm{d} x
\end{aligned}
$$

which rewrites, in terms of $v_{n}$ defined in (3.10), as

$$
\begin{aligned}
0=\frac{1}{4} \int_{0}^{T} h_{1 / 2, n} v_{n}^{\prime} z_{1, n}^{\prime} \mathrm{d} t+\int_{0}^{T} v_{n} \frac{z_{1, n}}{h_{1 / 2, n}} \mathrm{~d} t & \\
& +\int_{0}^{1}\left(\mathbb{R}_{\mathcal{S}_{n}} y_{n}^{1}\right)\left(\partial_{x} \mathbb{P}_{\mathcal{S}_{n}} z_{n}(0)\right) \mathrm{d} x-\int_{0}^{1}\left(\mathbb{Q}_{\mathcal{S}_{n}} y_{n}^{0}\right)\left(\mathbb{Q}_{\mathcal{S}_{n}} z_{n}^{\prime}(0)\right) \mathrm{d} x
\end{aligned}
$$

Now, consider $y_{n}$ the solution of (3.5) with boundary control $v_{n}$. Multiplying (3.5) by $z_{n}$ solution of (3.9) with initial data $\left(z_{n}^{0}, z_{n}^{1}\right)$, we get, after tedious computations that are left to the reader, that

$$
\begin{aligned}
0=\frac{1}{4} \int_{0}^{T} h_{1 / 2, n} v_{n}^{\prime} z_{1, n}^{\prime} \mathrm{d} t & +\int_{0}^{T} v_{n} \frac{z_{1, n}}{h_{1 / 2, n}} \mathrm{~d} t \\
& +\int_{0}^{1}\left(\mathbb{R}_{\mathcal{S}_{n}} y_{n}^{1}\right)\left(\partial_{x} \mathbb{P}_{\mathcal{S}_{n}} z_{n}(0)\right) \mathrm{d} x-\int_{0}^{1}\left(\mathbb{Q}_{\mathcal{S}_{n}} y_{n}^{0}\right)\left(\mathbb{Q}_{\mathcal{S}_{n}} z_{n}^{\prime}(0)\right) \mathrm{d} x \\
& \quad-\int_{0}^{1}\left(\mathbb{R}_{\mathcal{S}_{n}} y_{n}^{\prime}(T)\right)\left(\partial_{x} \mathbb{P}_{\mathcal{S}_{n}} z_{n}^{0}\right) \mathrm{d} x+\int_{0}^{1}\left(\mathbb{Q}_{\mathcal{S}_{n}} y_{n}(T)\right)\left(\mathbb{Q}_{\mathcal{S}_{n}} z_{n}^{1}\right) \mathrm{d} x
\end{aligned}
$$

Combined with (3.13), this yields that the solution $y_{n}$ of (3.5) satisfies the following property: For any $\left(z_{n}^{0}, z_{n}^{1}\right)$,

$$
-\int_{0}^{1}\left(\mathbb{R}_{\mathcal{S}_{n}} y_{n}^{\prime}(T)\right)\left(\partial_{x} \mathbb{P}_{\mathcal{S}_{n}} z_{n}^{0}\right) \mathrm{d} x+\int_{0}^{1}\left(\mathbb{Q}_{\mathcal{S}_{n}} y_{n}(T)\right)\left(\mathbb{Q}_{\mathcal{S}_{n}} z_{n}^{1}\right) \mathrm{d} x=0 .
$$

This obviously implies (3.6).

It is natural to ask if the discrete controls $v_{n}$ constructed in Lemma 3.1 converge to an admissible control for (3.1) under some assumptions on the convergence of $\left(y_{n}^{0}, y_{n}^{1}\right)$. We will prove that this is indeed the case. 
Given a sequence of meshes $\left(\mathcal{S}_{n}\right)_{n}$, we say that the sequence of discrete data $\left(a_{n}, b_{n}\right)_{n}$ defined on the meshes $\mathcal{S}_{n}$ strongly converges to $(a, b)$ in $L^{2}(0,1) \times H^{-1}(0,1)$ if:

$$
\mathbb{Q}_{\mathcal{S}_{n}} a_{n} \rightarrow a \quad \text { in } L^{2}(0,1), \quad \text { and } \quad \mathbb{R}_{\mathcal{S}_{n}} b_{n} \rightarrow\left(x \mapsto \int_{x}^{1} b(s) \mathrm{d} s\right) \quad \text { in } L^{2}(0,1) .
$$

Remark that this definition makes sense, since for $b \in H^{-1}(0,1)$, classical arguments allow to define the function $x \mapsto \int_{x}^{1} b(s) \mathrm{d} s$ in $L^{2}(0,1)$.

Theorem 3.2. Let $\left(y^{0}, y^{1}\right) \in L^{2}(0,1) \times H^{-1}(0,1)$ and $T>2$.

Given $M \geq 1$, we consider a sequence $\left(\mathcal{S}_{n}\right)$ of $M$-regular meshes, and a sequence of initial data $\left(y_{n}^{0}, y_{n}^{1}\right)$ which strongly converges to $\left(y^{0}, y^{1}\right)$ in $L^{2}(0,1) \times H^{-1}(0,1)$ in the sense of $(3.15)$.

Then the sequence of discrete controls $\left(v_{n}\right)_{n}$ given by Lemma 3.1 strongly converges in $L^{2}(0, T)$ to the HUM control $v_{\text {HUM }}$ for (3.1) with initial data $\left(y^{0}, y^{1}\right)$.

First of all, let us mention that, given $\left(y^{0}, y^{1}\right) \in L^{2}(0,1) \times H^{-1}(0,1)$, it is possible to find a sequence of initial data $\left(y_{n}^{0}, y_{n}^{1}\right)$ which strongly converges to $\left(y^{0}, y^{1}\right)$ in $L^{2}(0,1) \times H^{-1}(0,1)$ in the sense of $(3.15)$. We will briefly explain later (Rem. 3.5 below) how this can be done.

The proof of Theorem 3.2 is mainly based on inequality (1.11), that implies that the discrete controls $v_{n}$ are bounded in $L^{2}(0, T)$. Once this is proved, the result can be deduced from classical convergence properties of the scheme.

Proof. The proof is divided into several steps. First, we prove uniform bounds on the sequence $v_{n}$. Second, we prove that any weak limit of $v_{n}$ is an admissible control for (3.1). Third, we prove that there is only one weak limit, which coincides with the HUM-control $v_{\mathrm{HUM}}$ of (3.1). We finally prove the strong convergence of the controls $v_{n}$ in $L^{2}(0, T)$.

Uniform bounds. Since $\mathcal{J}_{n}\left(Z_{n}^{0}, Z_{n}^{1}\right) \leq \mathcal{J}_{n}(0,0)=0$, we have that

$$
\frac{1}{8} \int_{0}^{T} \rho(t)\left|Z_{1, n}^{\prime}\right|^{2}(t) \mathrm{d} t+\frac{1}{2} \int_{0}^{T}\left(\frac{Z_{1, n}(t)}{h_{1 / 2, n}}\right)^{2} \mathrm{~d} t \leq \sqrt{2 E_{* n}(0)} \sqrt{\left\|\mathbb{R}_{\mathcal{S}_{n}} y_{n}^{1}\right\|_{L^{2}(0,1)}^{2}+\left\|\mathbb{Q}_{\mathcal{S}_{n}} y_{n}^{0}\right\|_{L^{2}(0,1)}^{2}},
$$

where $E_{* n}(t)$ denotes the energy of $Z_{n}(t)$, which is constant. In view of the definition of $\rho$, since we assume that the meshes $\mathcal{S}_{n}$ are $M$-regular, inequality (1.11) holds. This, combined with the fact that $\left(\mathbb{Q}_{\mathcal{S}_{n}} y_{n}^{0}\right)$ and $\left(\mathbb{R}_{\mathcal{S}_{n}} y_{n}^{1}\right)$ are convergent in $L^{2}(0,1)$ and therefore bounded, leads us to

$$
k_{T} E_{* n}(T) \leq \frac{1}{8} \int_{0}^{T} \rho(t)\left|Z_{1, n}^{\prime}\right|^{2}(t) \mathrm{d} t+\frac{1}{2} \int_{0}^{T}\left(\frac{Z_{1, n}(t)}{h_{1 / 2, n}}\right)^{2} \mathrm{~d} t \leq C .
$$

Besides, multiplying (3.10) by $h_{1 / 2, n} v_{n}$ and integrating in time gives

$$
\begin{aligned}
\int_{0}^{T} \frac{h_{1 / 2, n}^{2}}{4}\left|v_{n}^{\prime}(t)\right|^{2}+\left|v_{n}(t)\right|^{2} \mathrm{~d} t=\int_{0}^{T}\left(\frac{h_{1 / 2, n}}{4} \rho(t) Z_{1, n}^{\prime}(t) v_{n}^{\prime}(t)+\frac{Z_{1, n}(t)}{h_{1 / 2, n}} v_{n}(t)\right) \mathrm{d} t \\
\quad \leq\left(\int_{0}^{T} \frac{h_{1 / 2, n}^{2}}{4}\left|v_{n}^{\prime}(t)\right|^{2}+\left|v_{n}(t)\right|^{2} \mathrm{~d} t\right)^{1 / 2}\left(\int_{0}^{T} \frac{\rho(t)}{4}\left|Z_{1, n}^{\prime}\right|^{2}(t) \mathrm{d} t+\int_{0}^{T}\left(\frac{Z_{1, n}(t)}{h_{1 / 2, n}}\right)^{2} \mathrm{~d} t\right)^{1 / 2}
\end{aligned}
$$

and therefore we obtain

$$
\int_{0}^{T} \frac{h_{1 / 2, n}^{2}}{4}\left|v_{n}^{\prime}(t)\right|^{2}+\left|v_{n}(t)\right|^{2} \mathrm{~d} t \leq C .
$$


We have thus proved, using the $M$-regularity assumption, that the sequence of discrete controls $v_{n}$ is bounded in $L^{2}(0, T)$. Therefore there exists a function $v \in L^{2}(0, T)$ such that

$$
v_{n} \rightarrow v, \quad \text { in } L^{2}(0, T) \text { weak, and } h_{1 / 2, n} v_{n}^{\prime} \rightarrow 0, \quad \text { in } L^{2}(0, T) \text { weak. }
$$

The second statement in (3.19) comes from the continuity of the derivation in the sense of distributions.

The function $v$ is an admissible control for (3.1). We need the following classical lemma on the convergence of the numerical schemes (which can be found for instance in [7]):

Lemma 3.3. Consider two smooth functions $\left(u^{0}, u^{1}\right)$ on $(0,1)$ such that $u^{0}(0)=u^{0}(1)=0$ and $u(x, t)$ the solution of the conservative system (1.1) with initial data $\left(u^{0}, u^{1}\right)$.

Given a sequence $\left(\mathcal{S}_{n}\right)_{n}$ of $M$-regular meshes, for all $n \in \mathbb{N}$, we denote by $u_{n}(t)$ the solution of the conservative semi-discrete scheme (1.7) with initial data

$$
u_{j, n}^{0}=u^{0}\left(x_{j, n}\right), \quad u_{j, n}^{1}=u^{1}\left(x_{j, n}\right), \quad j \in\{1, \ldots, n\} .
$$

Then $\left(\mathbb{P}_{\mathcal{S}_{n}} u_{j, n}, \mathbb{Q}_{\mathcal{S}_{n}} u_{j, n}^{\prime}\right)$ strongly converges to $\left(u, u^{\prime}\right)$ in $C\left([0, T] ; H_{0}^{1}(0,1) \times L^{2}(0,1)\right)$ and

$$
\frac{u_{1, n}(t)}{h_{1 / 2, n}} \rightarrow \partial_{x} u(0, t) \text { in } L^{2}(0, T), \quad \text { and } \quad u_{1, n}^{\prime}(t) \rightarrow 0 \text { in } L^{2}(0, T) .
$$

This result is of course still true for the backward system (3.4) and its semi-discrete approximations (3.9).

Now, consider two smooth functions $\left(z^{0}, z^{1}\right)$, and define, as in Lemma 3.3, the solution $z$ of the backward wave equation (3.4) with initial data $\left(z^{0}, z^{1}\right)$, and the solution $z_{n}$ of the semi-discrete systems (3.9), with initial data $\left(z^{0}\left(x_{j, n}\right), z^{1}\left(x_{j, n}\right)\right)$.

Using (3.19) and Lemma 3.3, we can pass to the limit in (3.13) and obtain that the solution $z$ of (3.4) satisfies:

$$
0=\int_{0}^{T} v(t) \partial_{x} z(0, t) \mathrm{d} t+\left\langle y^{1}, z(., 0)\right\rangle_{H^{-1}(0,1) \times H_{0}^{1}(0,1)}-\int_{0}^{1} y^{0}(x) \partial_{t} z(x, 0) \mathrm{d} x .
$$

By a density argument, this identity can be extended to any $\left(z^{0}, z^{1}\right) \in H_{0}^{1}(0,1) \times L^{2}(0, T)$.

Besides, for any $\left(z^{0}, z^{1}\right) \in H_{0}^{1}(0,1) \times L^{2}(0,1)$, as in (3.14), multiplying the solution of (3.1) with boundary condition $y(0, t)=v(t)$ and initial data $\left(y^{0}, y^{1}\right)$ by $z$ solution of (3.4) with initial data $\left(z^{0}, z^{1}\right)$, we obtain that

$$
\begin{aligned}
0=\int_{0}^{T} v(t) \partial_{x} z(0, t) \mathrm{d} t+\left\langle y^{1}, z(., 0)\right\rangle_{H^{-1}(0,1) \times H_{0}^{1}(0,1)}-\int_{0}^{1} & y^{0}(x) \partial_{t} z(x, 0) \mathrm{d} x \\
& \quad-\left\langle\partial_{t} y(T), z^{0}\right\rangle_{H^{-1}(0,1) \times H_{0}^{1}(0,1)}+\int_{0}^{1} y(T, x) z^{1}(x) \mathrm{d} x .
\end{aligned}
$$

Hence we deduce from (3.21) that

$$
\left\langle\partial_{t} y(T), z^{0}\right\rangle_{H^{-1}(0,1) \times H_{0}^{1}(0,1)}-\int_{0}^{1} y(T, x) z^{1}(x) \mathrm{d} x=0 .
$$

Therefore $y$ satisfies (3.2). This precisely means that $v$ is an admissible control for (3.1).

The limit $v$ is the HUM control $v_{\mathrm{HUM}}$. It is sufficient to prove that $v(t)$ coincides with some $\partial_{x} z(t, 0)$, where $z$ is the solution of (3.4) for some initial data $\left(z^{0}, z^{1}\right) \in H_{0}^{1}(0,1) \times L^{2}(0,1)$, see for instance [21].

From (3.16), there exist two functions $Z^{0} \in H_{0}^{1}(0,1)$ and $Z^{1} \in L^{2}(0,1)$ such that

$$
\mathbb{P}_{\mathcal{S}_{n}} Z_{n}^{0} \rightarrow Z^{0}, H_{0}^{1}(0,1) \text { weak, and } \mathbb{Q}_{\mathcal{S}_{n}} Z_{n}^{1} \rightarrow Z^{1}, L^{2}(0,1) \text { weak } .
$$


Using the weak formulations of (3.9) and the conservation of the energy, we can prove (the proof can be adapted in a standard way from the arguments in [7], and in particular from Lem. 3.3 stated above, and is left to the reader) that:

$$
\begin{aligned}
& \left(\mathbb{P}_{\mathcal{S}_{n}} Z_{n}, \mathbb{Q}_{\mathcal{S}_{n}} Z_{n}\right) \rightarrow\left(Z, Z^{\prime}\right) \quad \text { in } L^{\infty}\left(0, T ; H_{0}^{1}(0,1) \times L^{2}(0,1)\right) * \text { weak } \\
& \forall t \in[0, T], \quad\left(\mathbb{P}_{\mathcal{S}_{n}} Z_{n}(t), \mathbb{Q}_{\mathcal{S}_{n}} Z_{n}(t)\right) \rightarrow\left(Z(t), Z^{\prime}(t)\right) \quad \text { in } H_{0}^{1}(0,1) \times L^{2}(0,1) \text { weak, }
\end{aligned}
$$

where $Z$ is the solution of (3.4) with initial data $\left(Z^{0}, Z^{1}\right)$. Besides, one easily shows that

$$
\frac{Z_{1, n}}{h_{1 / 2, n}}-\frac{h_{1 / 2, n}}{4} Z_{1, n}^{\prime \prime} \rightarrow \partial_{x} Z(0, t), \quad \text { in } \quad \mathcal{D}^{\prime}(0, T) .
$$

But $Z_{1, n} / h_{1 / 2, n}$ is bounded in $L^{2}(0, T)$ from (3.16), and therefore $h_{1 / 2, n} Z_{1, n}^{\prime \prime} \rightarrow 0$ in $\mathcal{D}^{\prime}(0, T)$. This also gives that

$$
\frac{Z_{1, n}}{h_{1 / 2, n}} \rightarrow \partial_{x} Z \quad \text { in } \mathcal{D}^{\prime}(0, T), \quad Z_{1, n} \rightarrow 0 \quad \text { in } \mathcal{D}^{\prime}(0, T), \quad h_{1 / 2, n}\left(\rho Z_{1, n}^{\prime}\right)^{\prime} \rightarrow 0 \quad \text { in } \mathcal{D}^{\prime}(0, T)
$$

Combined with the definition of $v_{n}$ in Lemma 3.1, it follows that

$$
-\frac{h_{1 / 2, n}^{2}}{4} v_{n}^{\prime \prime}+v_{n} \rightarrow \partial_{x} Z(0, t), \text { in } \mathcal{D}^{\prime}(0, T) .
$$

But, since $v_{n}$ is bounded in $L^{2}(0, T)$ by (3.18),

$$
h_{1 / 2, n}^{2} v_{n}^{\prime \prime} \rightarrow 0 \text { in } \mathcal{D}^{\prime}(0, T),
$$

and therefore $v(t)=\partial_{x} Z(0, t)$ in $\mathcal{D}^{\prime}(0, T)$.

Since we have already proved that $v$ is an admissible control for (1.1), this proves that $v$ is the HUM control $v_{\text {HUM }}$.

Strong convergence. Since the weak convergence is already proven, it is sufficient to prove the convergence of the $L^{2}(0, T)$-norms.

Since $v(t)=\partial_{x} Z(0, t)$ for a solution $Z$ of (3.4) with initial data $\left(Z^{0}, Z^{1}\right)$, we get from (3.21) that:

$$
0=\int_{0}^{T}\left(\partial_{x} Z(0, t)\right)^{2} \mathrm{~d} t+\left\langle y^{1}, Z(., 0)\right\rangle_{H^{-1}(0,1) \times H_{0}^{1}(0,1)}-\int_{0}^{1} y^{0}(x) \partial_{t} Z(x, 0) \mathrm{d} x .
$$

But (3.13) gives:

$$
\begin{aligned}
0=\frac{1}{4} \int_{0}^{T} \rho(t)\left|Z_{1, n}^{\prime}(t)\right|^{2} \mathrm{~d} t & +\int_{0}^{T}\left|\frac{Z_{1, n}(t)}{h_{1 / 2, n}}\right|^{2} \mathrm{~d} t \\
& +\int_{0}^{1}\left(\mathbb{R}_{\mathcal{S}_{n}} y_{n}^{1}\right)(x) \partial_{x}\left(\mathbb{P}_{\mathcal{S}_{n}} Z_{n}\right)(x, 0) \mathrm{d} x-\int_{0}^{1}\left(\mathbb{Q}_{\mathcal{S}_{n}} y_{n}^{0}\right)(x)\left(\mathbb{Q}_{\mathcal{S}_{n}} Z_{n}^{\prime}\right)(x, 0) \mathrm{d} x .
\end{aligned}
$$

Convergences (3.22) and (3.15) imply that we can pass to the limit in the linear term, and therefore, by (3.25), we get:

$$
\frac{1}{4} \int_{0}^{T} \rho(t)\left|Z_{1, n}^{\prime}(t)\right|^{2} \mathrm{~d} t+\int_{0}^{T}\left|\frac{Z_{1, n}(t)}{h_{1 / 2, n}}\right|^{2} \mathrm{~d} t \rightarrow \int_{0}^{T}\left|\partial_{x} Z(0, t)\right|^{2} \mathrm{~d} t
$$


Combined with the weak convergences (3.24), this proves the following strong convergences:

$$
\left\{\begin{array}{l}
\sqrt{\rho} Z_{1, n}^{\prime} \rightarrow 0, \\
\frac{Z_{1, n}}{h_{1 / 2, n}}(t) \rightarrow \partial_{x} Z(0, t), \quad \text { in } L^{2}(0, T) .
\end{array}\right.
$$

But, from the definition (3.10) of $v_{n}$, the convergence (3.19) implies that:

$$
\begin{aligned}
\int_{0}^{T} \frac{h_{1 / 2, n}^{2}}{4}\left|v_{n}^{\prime}(t)\right|^{2}+\left|v_{n}(t)\right|^{2} \mathrm{~d} t=\int_{0}^{T} \frac{h_{1 / 2, n}}{4} \rho(t) Z_{1, n}^{\prime}(t) v_{n}^{\prime}(t)+\frac{Z_{1, n}(t)}{h_{1 / 2, n}} v_{n}(t) \mathrm{d} t & \\
& \longrightarrow \int_{0}^{T} \partial_{x} Z(0, t) v(t) \mathrm{d} t=\int_{0}^{T} v(t)^{2} \mathrm{~d} t .
\end{aligned}
$$

Hence we deduce from (3.19) that:

$$
h_{1 / 2, n} v_{n}^{\prime} \rightarrow 0 \quad \text { in } L^{2}(0, T), \quad \text { and } \quad v_{n} \rightarrow v=v_{H U M} \quad \text { in } L^{2}(0, T),
$$

which concludes the proof of Theorem 3.2.

Remark 3.4. The proof of Theorem 3.2 slightly differs from the one in [5], which presented an approach based on the spectral decomposition of the solutions. This technique, in our context, seems more technically involved than the one presented above, since the spectrum is not as explicit as in the case of a uniform mesh.

Remark 3.5. Let us briefly comment the hypothesis (3.15), and prove that, given $(a, b) \in L^{2}(0,1) \times H^{-1}(0,1)$ and a sequence $\mathcal{S}_{n}$ of $M$-regular meshes, there exists a sequence of discrete data $\left(a_{n}, b_{n}\right)$ defined on the mesh $\mathcal{S}_{n}$ which strongly converges to $(a, b)$ in $L^{2}(0,1) \times H^{-1}(0,1)$ in the sense of $(3.15)$.

Indeed, for $a \in L^{2}(0,1)$, define $a_{n}=\mathbb{A}_{\mathcal{S}_{n}}(a)$ as follows (recall the convention $a_{n+1, n}=0$ ):

$$
a_{j, n}+a_{j+1, n}=\frac{2}{h_{j+1 / 2, n}} \int_{x_{j, n}}^{x_{j+1, n}} a(x) \mathrm{d} x, \quad 1 \leq j \leq n .
$$

If $a$ is continuous on $[0,1]$, one easily checks that

$$
\left\|\mathbb{Q}_{\mathcal{S}_{n}}\left(\mathbb{A}_{\mathcal{S}_{n}}(a)\right)-a\right\|_{L^{2}(0,1)} \rightarrow 0 .
$$

Besides, if $a$ is in $L^{2}$, we have that

$$
\left\|\mathbb{Q}_{\mathcal{S}_{n}}\left(\mathbb{A}_{\mathcal{S}_{n}}(a)\right)\right\|_{L^{2}(0,1)} \leq C\|a\|_{L^{2}(0,1)} .
$$

This, using the density of the continuous functions in $L^{2}(0,1)$, is sufficient to prove that the sequence of discrete data $a_{n}=\mathbb{Q}_{\mathcal{S}_{n}}\left(\mathbb{A}_{\mathcal{S}_{n}}(a)\right)$ converges to $a$ in $L^{2}(0,1)$ for all $a \in L^{2}(0,1)$.

For the approximation of $b \in H^{-1}(0,1)$, we look for an approximation of

$$
B(x)=\int_{x}^{1} b(s) \mathrm{d} s,
$$

which lies in $L^{2}(0,1)$. Thus, the sequence $B_{n}=\mathbb{A}_{\mathcal{S}_{n}} B$ provides discrete data which satisfy $\mathbb{Q}_{\mathcal{S}_{n}}\left(B_{n}\right) \rightarrow B$ in $L^{2}(0,1)$ when $n \rightarrow \infty$. It is then sufficient to find discrete data $b_{n}$ such that $\mathbb{R}_{\mathcal{S}_{n}} b_{n}=\mathbb{Q}_{\mathcal{S}_{n}} B_{n}$, and this can be done explicitly. 


\section{Application to the damped wave equation}

\subsection{The continuous setting}

We consider the continuous damped wave equation on the interval $(0,1)$ :

$$
\begin{cases}\partial_{t t}^{2} w-\partial_{x x}^{2} w+2 \sigma \partial_{t} w=0, & (x, t) \in(0,1) \times(0, \infty) \\ w(0, t)=w(1, t)=0, & t \in(0, \infty) \\ w(x, 0)=w^{0}(x), \quad \partial_{t} w(x, 0)=w^{1}(x), & x \in(0,1)\end{cases}
$$

with $w^{0} \in H_{0}^{1}(0,1)$ and $w^{1} \in L^{2}(0,1)$.

We assume that the damping function $\sigma=\sigma(x)$ is bounded, non-negative and bounded from below by a positive number on a subinterval $J$, that is there exists $\alpha>0$, such that

$$
\sigma(x) \geq \alpha, \quad \forall x \in J, \quad \text { and } \quad\|\sigma\|_{\infty}=K .
$$

Then the energy, defined by (1.2), satisfies the dissipation law

$$
\frac{\mathrm{d} E}{\mathrm{~d} t}(t)=-2 \int_{0}^{1} \sigma(x)\left|\partial_{t} w(t, x)\right|^{2} \mathrm{~d} x, \quad t \geq 0 .
$$

It is well-known that, under the assumption (4.2), the energy is exponentially decaying: There exist positive constants $C$ and $\mu$ such that

$$
E(t) \leq C E(0) \exp (-\mu t), \quad t \geq 0 .
$$

Using classical arguments in stabilization theory (see [16]), the energy of (4.1) is exponentially decaying if and only if the observability inequality (1.5) holds for solutions of the conservative system (1.1).

\subsection{The semi-discrete setting}

We consider a mesh $\mathcal{S}_{n}$ as in (1.6), and discretize equation (4.1) according to the mixed finite element method:

$$
\left\{\begin{array}{l}
\frac{h_{j-1 / 2, n}}{4}\left(w_{j-1, n}^{\prime \prime}+w_{j, n}^{\prime \prime}\right)+\frac{h_{j+1 / 2, n}}{4}\left(w_{j, n}^{\prime \prime}+w_{j+1, n}^{\prime \prime}\right)= \\
\quad-\frac{h_{j-1 / 2, n} \sigma_{j-1 / 2, n}}{2}\left(w_{j-1, n}^{\prime}+w_{j, n}^{\prime}\right)-\frac{h_{j+1 / 2, n} \sigma_{j+1 / 2, n}}{2}\left(w_{j, n}^{\prime}+w_{j+1, n}^{\prime}\right) \\
\quad+\frac{w_{j+1, n}-w_{j, n}}{h_{j+1 / 2, n}}-\frac{w_{j, n}-w_{j-1, n}}{h_{j-1 / 2, n}}, \quad j=1, \ldots, n, t \in[0, \infty), \\
w_{0, n}(t)=w_{n+1, n}(t)=0, \quad t \in[0, \infty), \\
w_{j, n}(0)=w_{j, n}^{0}, \quad w_{j, n}^{\prime}(0)=w_{j, n}^{1}, \quad j=1, \ldots, n,
\end{array}\right.
$$

where $\sigma_{j+1 / 2, n}$ is an approximation on $\left[x_{j, n}, x_{j+1, n}\right]$ of the damping function $\sigma$ in (4.1) which is assumed to satisfy the following properties:

$$
\sigma_{j+1 / 2, n} \geq \alpha, \quad \forall\left[x_{j, n}, x_{j+1, n}\right] \subset J, \quad \text { and } \quad 0 \leq \sigma_{j+1 / 2, n} \leq K, \quad \forall j \in\{0, \ldots, n\},
$$

where $\alpha, K$ and $J$ are as in (4.2).

The energy (1.8) of solutions of (4.5) satisfies

$$
\frac{\mathrm{d} E_{n}}{\mathrm{~d} t}(t)=-2 \sum_{j=0}^{n} h_{j+1 / 2, n} \sigma_{j+1 / 2, n}\left(\frac{w_{j, n}^{\prime}(t)+w_{j+1, n}^{\prime}(t)}{2}\right)^{2} .
$$


Obviously, this dissipation law corresponds to a discrete version of (4.3).

The question we investigate is the following: Given a sequence $\left(\mathcal{S}_{n}\right)_{n}$ of meshes, can we find positive constants $C$ and $\mu$ independent of $n$ such that

$$
E_{n}(t) \leq C E_{n}(0) \exp (-\mu t), \quad t \geq 0,
$$

for any solution of (4.5) on $\mathcal{S}_{n}$ ?

Similarly as in the continuous setting, this property is equivalent to the uniform observability inequality (1.12) for solutions of the conservative system (1.7) (see for instance [27]). Therefore Theorem 1.2 leads to the following result:

Theorem 4.1. Let $M \geq 1$, and consider a sequence $\left(\mathcal{S}_{n}\right)_{n}$ of $M$-regular meshes and a sequence of damping functions $\sigma_{n}$ satisfying (4.6).

Then there exist positive constants $C$ and $\mu$ such that for all $n$, inequality (4.8) holds for any solution of (4.5) on $\mathcal{S}_{n}$.

The proof of Theorem 4.1, which can be adapted in a standard way from [16] or [27], is left to the reader.

Remark 4.2. Note that this method yields an estimate on the decay rate $\mu$ appearing in (4.8), which is far from being optimal in general. This is a drawback of the method, which is based on a perturbation argument of the conservative system. Even in the continuous setting, the decay rate parameter obtained through this method is not in general the sharp one, which is known to coincide (at least in the one dimensional case) with the spectral abscissa (see [8]).

Remark 4.3. The analysis proposed here can be applied as well to the 1d Perfectly Matched Layers equations (see $[2,10])$, which, roughly, consists in a damped wave equation written in hyperbolic form:

$$
\begin{cases}\partial_{t} p+\partial_{x} q+\sigma p=0, & (x, t) \in(0,1) \times(0, \infty), \\ \partial_{t} q+\partial_{x} p+\sigma q=0, & (x, t) \in(0,1) \times(0, \infty), \\ q(0, t)=p(1, t)=0, & t \in(0, \infty), \\ q(x, 0)=q_{0}(x), \quad p(x, 0)=p_{0}(x), & x \in(0,1),\end{cases}
$$

where $\sigma$ satisfies the assumptions (4.2).

In [10], it is proven that the 1d PML system is exponentially stable: The energy of solutions of (4.9), defined as

is exponentially decaying.

$$
E(t)=\frac{1}{2} \int_{0}^{1}|p(t, x)|^{2}+|q(t, x)|^{2} \mathrm{~d} x,
$$

Besides, stabilization properties for space semi-discrete approximation schemes on uniform meshes are studied in [10]: It is proved that finite difference approximation schemes are not uniformly exponentially stable, but adding a viscosity term in space makes the schemes uniformly exponentially stable.

We claim that the so-called Box scheme (see for instance [4,13]) on $M$-regular meshes for the 1d PML equations also are exponentially stable. To be more precise, for $\mathcal{S}_{n}$ an $M$-regular mesh, we consider the space approximation scheme of (4.9) given by:

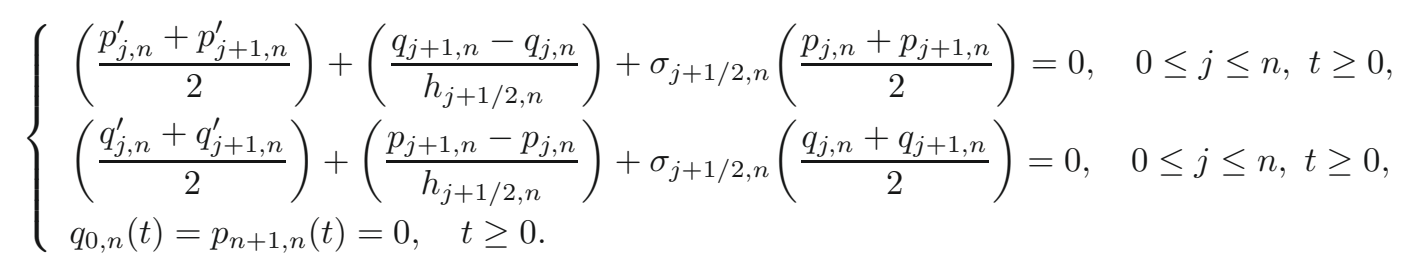


Then the energy of solutions $\left(p_{n}, q_{n}\right)$ of (4.10), defined by

$$
\begin{aligned}
& E_{n}(t)=\frac{1}{2} \sum_{j=0}^{n} h_{j+1 / 2, n}\left(\left(\frac{p_{j, n}+p_{j+1, n}}{2}\right)^{2}+\left(\frac{q_{j, n}+q_{j+1, n}}{2}\right)^{2}\right) \\
& +\frac{1}{8}\left(\frac{1}{n+1}\right)^{2} \sum_{j=0}^{n} h_{j+1 / 2, n}\left(\left(\frac{p_{j, n}^{\prime}+p_{j+1, n}^{\prime}}{2}\right)^{2}+\left(\frac{q_{j, n}^{\prime}+q_{j+1, n}^{\prime}}{2}\right)^{2}\right)
\end{aligned}
$$

is exponentially decaying, uniformly with respect to $n$. The proof of this result will be published elsewhere.

\section{Fully DisCRETE SCHEMeS}

In this section, we briefly explain how the results in $[11,12]$ can be combined with Theorem 1.2 to obtain observability and stabilization results in the fully discrete setting.

\subsection{Observability for fully discrete approximation schemes of (1.7)}

This subsection is based on the results in [12]. For $\Delta t>0$, with the same notations as in (1.7), we introduce the following fully discrete approximation scheme (corresponding to the midpoint scheme):

$$
\left\{\begin{array}{l}
\frac{h_{j-1 / 2, n}}{4}\left(\frac{u_{j-1, n}^{\tau+1}+u_{j-1, n}^{\tau-1}-2 u_{j-1, n}^{\tau}}{(\Delta t)^{2}}+\frac{u_{j, n}^{\tau+1}+u_{j, n}^{\tau-1}-2 u_{j, n}^{\tau}}{(\Delta t)^{2}}\right) \\
+\frac{h_{j+1 / 2, n}}{4}\left(\frac{u_{j, n}^{\tau+1}+u_{j, n}^{\tau-1}-2 u_{j, n}^{\tau}}{(\Delta t)^{2}}+\frac{u_{j+1, n}^{\tau+1}+u_{j+1, n}^{\tau-1}-2 u_{j+1, n}^{\tau}}{(\Delta t)^{2}}\right) \\
\quad=\frac{1}{h_{j+1 / 2, n}}\left(\frac{u_{j+1, n}^{\tau-1}+2 u_{j+1, n}^{\tau}+u_{j+1, n}^{\tau+1}}{4}-\frac{u_{j, n}^{\tau-1}+2 u_{j, n}^{\tau}+u_{j, n}^{\tau+1}}{4}\right) \\
\quad-\frac{1}{h_{j-1 / 2, n}}\left(\frac{u_{j, n}^{\tau-1}+2 u_{j, n}^{\tau}+u_{j, n}^{\tau+1}}{4}-\frac{u_{j-1, n}^{\tau-1}+2 u_{j-1, n}^{\tau}+u_{j-1, n}^{\tau+1}}{4}\right), j=1, \ldots, n, \tau \in \mathbb{N}, \\
u_{0, n}^{\tau}=u_{n+1, n}^{\tau}=0, \quad \tau \in \mathbb{N}, \quad\left(\frac{u_{j, n}^{1}-u_{j, n}^{0}}{\Delta t}\right)=V_{j, n}, \quad j=1, \ldots, n .
\end{array}\right.
$$

In (5.1), $u_{j, n}^{\tau}$ stands for an approximation of the solution $u_{n}$ of (1.7) at the point $x_{j, n}$ and time $t_{\tau}=\tau \Delta t$.

The energy of solutions of (5.1), defined by

$$
\begin{aligned}
E_{n}^{\tau+1 / 2}=\frac{1}{2} \sum_{j=0}^{n} h_{j+1 / 2, n} & {\left[\frac{1}{h_{j+1 / 2, n}}\left(\frac{u_{j+1, n}^{\tau}+u_{j+1, n}^{\tau+1}}{2}-\frac{u_{j, n}^{\tau}+u_{j, n}^{\tau+1}}{2}\right)\right]^{2} } \\
& +\frac{1}{2} \sum_{j=0}^{n} h_{j+1 / 2, n}\left[\frac{1}{\Delta t}\left(\frac{u_{j, n}^{\tau+1}+u_{j+1, n}^{\tau+1}}{2}-\frac{u_{, n}^{\tau}+u_{j+1, n}^{\tau}}{2}\right)\right]^{2},
\end{aligned}
$$

is independent of $\tau \in \mathbb{N}$.

Introduce, for $s>0$, the filtered class

$$
\mathcal{C}_{n}(s)=\operatorname{span}\left\{\phi_{n}^{k} \text { such that the corresponding eigenvalue } \lambda_{n}^{k} \text { satisfies }\left|\lambda_{n}^{k}\right| \leq s\right\} .
$$


Then the results in [12] apply. Combined with Theorem 1.2, they yield:

Theorem 5.1. Let $M$ be a real number greater than one, and consider a sequence $\left(\mathcal{S}_{n}\right)_{n}$ of $M$-regular meshes. Let $\delta$ be a positive number.

Then there exist a time $T$ and positive constants $k_{T}$ and $K_{T}$ such that for all integer $n$ and $\Delta t>0$, any solution $u_{n}$ of (5.1) with initial data

$$
\left(U_{n}, V_{n}\right) \in \mathcal{C}_{n}(\delta / \Delta t)^{2}
$$

satisfies

$$
k_{T} E_{n}^{1 / 2} \leq \Delta t \sum_{\tau \Delta t \in[0, T]}\left[\left(\frac{u_{1, n}^{\tau}+u_{1, n}^{\tau+1}}{2 h_{1 / 2, n}}\right)^{2}+\left(\frac{u_{1, n}^{\tau+1}-u_{1, n}^{\tau}}{\Delta t}\right)^{2}\right] \leq K_{T} E_{n}^{1 / 2} .
$$

Besides, if $J=(a, b) \subset(0,1)$ denotes a subinterval of $(0,1)$, then there exist a time $T$ and a constant $C_{T}$ such that for all integer $n$ and $\Delta t>0$, any solution $u_{n}$ of (5.1) with initial data $\left(U_{n}, V_{n}\right)$ satisfying (5.3) satisfies

$$
E_{n}^{1 / 2} \leq C_{T} \Delta t \sum_{\tau \Delta t \in[0, T]} \sum_{x_{j, n} \in J} h_{j+1 / 2, n}\left[\frac{1}{\Delta t}\left(\frac{u_{j, n}^{\tau+1}+u_{j+1, n}^{\tau+1}}{2}-\frac{u_{, n}^{\tau}+u_{j+1, n}^{\tau}}{2}\right)\right]^{2}
$$

Note that if the CFL type condition

$$
(n+1)^{2}(\Delta t) \leq \frac{\delta \pi}{4 M}
$$

is satisfied, then condition (5.3) is always satisfied due to (2.11), and then no filtering condition is required for estimates (5.4) to hold.

Note that the filtering condition (5.3) is needed for having uniform observability results. This will be proved elsewhere.

Also remark that the results in [12] apply to a wider class of time-discretization schemes. We refer the interested reader to [12] for precise statements.

As in the time continuous case, Theorem 5.1 can be applied to obtain convergence of the discrete controls computed on the fully discrete schemes toward the HUM control of the continuous system, provided that, for some $\delta>0$, the CFL type condition (5.6) is always satisfied when $\Delta t \rightarrow 0, n \rightarrow \infty$.

\subsection{Stabilization properties}

This section is based on the recent article [11], where stabilization issues were discussed for fully discrete versions of abstract damped systems such as (4.5). Roughly speaking, the results in [11] follow the investigation in [12], and present two different cases:

- When the space and time discretization parameters are not related, one needs to add a numerical viscosity term in time at scale $1 / \Delta t$ to efficiently damp out the high-frequency components which cannot be observed on the corresponding conservative fully discrete equations (5.1) (see Thm. 5.1).

- When a CFL type condition similar to (5.6) is satisfied, the natural fully discrete scheme associated to (4.5) indeed are uniformly exponentially stable, with respect to both space and time discretization parameters. 
We first rewrite equations (4.5) as a system of two first order in time equations, by setting $v_{j, n}=w_{j, n}^{\prime}$ in (4.5). The full system then writes $w_{j, n}^{\prime}=v_{j, n}$ and

$$
\left\{\begin{array}{c}
\frac{h_{j-1 / 2, n}}{4}\left(v_{j-1, n}^{\prime}+v_{j, n}^{\prime}\right)+\frac{h_{j+1 / 2, n}}{4}\left(v_{j, n}^{\prime}+v_{j+1, n}^{\prime}\right)= \\
-\frac{h_{j-1 / 2, n} \sigma_{j-1 / 2, n}}{2}\left(v_{j-1, n}+v_{j, n}\right)-\frac{h_{j+1 / 2, n} \sigma_{j+1 / 2, n}}{2}\left(v_{j, n}+v_{j+1, n}\right) \\
+\frac{w_{j+1, n}-w_{j, n}}{h_{j+1 / 2, n}}-\frac{w_{j, n}-w_{j-1, n}}{h_{j-1 / 2, n}}, \quad j=1, \ldots, n, t \in[0, \infty), \\
w_{0, n}(t)=w_{n+1, n}(t)=v_{0, n}(t)=v_{n+1, n}(t)=0, \quad t \in[0, \infty), \\
w_{j, n}(0)=w_{j, n}^{0}, \quad v_{j, n}(0)=w_{j, n}^{1}, \quad j=1, \ldots, n .
\end{array}\right.
$$

We then discretize in time these equations according to the midpoint scheme:

$$
\left\{\begin{array}{l}
\frac{\tilde{w}_{j, n}^{\tau+1}-w_{j, n}^{\tau}}{\Delta t}=\frac{v_{j, n}^{\tau}+\tilde{v}_{j, n}^{\tau+1}}{2}, \\
\frac{h_{j-1 / 2, n}}{4(\Delta t)}\left(\left(\tilde{v}_{j-1, n}^{\tau+1}+\tilde{v}_{j, n}^{\tau+1}\right)-\left(v_{j-1, n}^{\tau}+v_{j, n}^{\tau}\right)\right)+\frac{h_{j+1 / 2, n}}{4(\Delta t)}\left(\left(\tilde{v}_{j, n}^{\tau+1}+\tilde{v}_{j+1, n}^{\tau+1}\right)-\left(v_{j, n}^{\tau}+v_{j+1, n}^{\tau}\right)\right) \\
=\frac{1}{2 h_{j+1 / 2, n}}\left(\left(w_{j+1, n}^{\tau}+\tilde{w}_{j+1, n}^{\tau+1}\right)-\left(w_{j, n}^{\tau}+\tilde{w}_{j, n}^{\tau+1}\right)\right)-\frac{1}{2 h_{j-1 / 2, n}}\left(\left(w_{j, n}^{\tau}+\tilde{w}_{j, n}^{\tau+1}\right)-\left(w_{j-1, n}^{\tau}+\tilde{w}_{j-1, n}^{\tau+1}\right)\right) \\
\quad-\frac{h_{j-1 / 2, n}}{4} \sigma_{j-1 / 2, n}\left(\left(v_{j-1, n}^{\tau}+\tilde{v}_{j-1, n}^{\tau+1}\right)+\left(v_{j, n}^{\tau}+\tilde{v}_{j, n}^{\tau+1}\right)\right) \\
\quad-\frac{h_{j+1 / 2, n}}{4} \sigma_{j+1 / 2, n}\left(\left(v_{j, n}^{\tau}+\tilde{v}_{j, n}^{\tau+1}\right)+\left(v_{j+1, n}^{\tau}+\tilde{v}_{j+1, n}^{\tau+1}\right)\right), \quad j=1, \ldots, n, \tau \in \mathbb{N} .
\end{array}\right.
$$

One then needs to complete this set of equations with a relation between the quantities $\left(\tilde{w}^{\tau+1}, \tilde{v}^{\tau+1}\right)$ and $\left(w^{\tau+1}, v^{\tau+1}\right)$. We will consider two different ways of doing this:

- With a numerical viscosity term:

$$
\left\{\begin{array}{l}
\frac{h_{j-1 / 2, n}}{4(\Delta t)}\left(\left(w_{j-1, n}^{\tau+1}+w_{j, n}^{\tau+1}\right)-\left(\tilde{w}_{j-1, n}^{\tau+1}+\tilde{w}_{j, n}^{\tau+1}\right)\right)+\frac{h_{j+1 / 2, n}}{4(\Delta t)}\left(\left(w_{j, n}^{\tau+1}+w_{j+1, n}^{\tau+1}\right)-\left(\tilde{w}_{j, n}^{\tau+1}+\tilde{w}_{j, n}^{\tau+1}\right)\right) \\
=(\Delta t)^{2}\left(\frac{w_{j+1, n}^{\tau+1}-w_{j, n}^{\tau+1}}{h_{j+1 / 2, n}}-\frac{w_{j, n}^{\tau+1}-w_{j-1, n}^{\tau+1}}{h_{j-1 / 2, n}}\right), \quad j=1, \ldots, n, \tau \in \mathbb{N}, \\
\frac{h_{j-1 / 2, n}}{4(\Delta t)}\left(\left(v_{j-1, n}^{\tau+1}+v_{j, n}^{\tau+1}\right)-\left(\tilde{v}_{j-1, n}^{\tau+1}+\tilde{v}_{j, n}^{\tau+1}\right)\right)+\frac{h_{j+1 / 2, n}}{4(\Delta t)}\left(\left(v_{j, n}^{\tau+1}+v_{j+1, n}^{\tau+1}\right)-\left(\tilde{v}_{j, n}^{\tau+1}+\tilde{v}_{j, n}^{\tau+1}\right)\right) \\
=(\Delta t)^{2}\left(\frac{v_{j+1, n}^{\tau+1}-v_{j, n}^{\tau+1}}{h_{j+1 / 2, n}}-\frac{v_{j, n}^{\tau+1}-v_{j-1, n}^{\tau+1}}{h_{j-1 / 2, n}}\right), \quad j=1, \ldots, n, \tau \in \mathbb{N} .
\end{array}\right.
$$

- Without any numerical viscosity term:

$$
w_{j, n}^{\tau+1}=\tilde{w}_{j, n}^{\tau+1}, \quad v_{j, n}^{\tau+1}=\tilde{v}_{j, n}^{\tau+1}, \quad j=1, \ldots, n, \tau \in \mathbb{N} .
$$

In both situations, we impose the boundary conditions

$$
w_{0, n}^{\tau}=w_{n+1, n}^{\tau}=v_{0, n}^{\tau}=v_{n+1, n}^{\tau}=\tilde{w}_{0, n}^{\tau}=\tilde{w}_{n+1, n}^{\tau}=\tilde{v}_{0, n}^{\tau}=\tilde{v}_{n+1, n}^{\tau}=0, \quad \tau \in \mathbb{N} .
$$


The energy of solutions of (5.7)-(5.8)-(5.10) (or of (5.7)-(5.9)-(5.10)), given by

$$
\begin{cases}E_{n}^{\tau}=\frac{1}{2} \sum_{j=0}^{n} h_{j+1 / 2, n}\left(\frac{w_{j+1, n}^{\tau}-w_{j, n}^{\tau}}{h_{j+1 / 2, n}}\right)^{2}+\frac{1}{2} \sum_{j=0}^{n} h_{j+1 / 2, n}\left(\frac{v_{j, n}^{\tau}+v_{j+1, n}^{\tau}}{2}\right)^{2}, & \tau \in \mathbb{N}, \\ \tilde{E}_{n}^{\tau}=\frac{1}{2} \sum_{j=0}^{n} h_{j+1 / 2, n}\left(\frac{\tilde{w}_{j+1, n}^{\tau}-\tilde{w}_{j, n}^{\tau}}{h_{j+1 / 2, n}}\right)^{2}+\frac{1}{2} \sum_{j=0}^{n} h_{j+1 / 2, n}\left(\frac{\tilde{v}_{j, n}^{\tau}+\tilde{v}_{j+1, n}^{\tau}}{2}\right)^{2}, & \tau \in \mathbb{N},\end{cases}
$$

is decaying, since one can check that, in both cases,

$$
\left\{\begin{array}{l}
\frac{\tilde{E}_{n}^{\tau+1}-E_{n}^{\tau}}{\Delta t}=-2 \sum_{j=0}^{n} h_{j+1 / 2, n} \sigma_{j+1 / 2, n}\left(\frac{v_{j, n}^{\tau}+v_{j+1, n}^{\tau}+\tilde{v}_{j, n}^{\tau+1}+\tilde{v}_{j+1, n}^{\tau+1}}{4}\right)^{2}, \quad \tau \in \mathbb{N}, \\
\frac{E_{n}^{\tau+1}-\tilde{E}_{n}^{\tau+1}}{\Delta t} \leq 0, \quad \tau \in \mathbb{N} .
\end{array}\right.
$$

Let us briefly analyze the equations (5.7)-(5.8)-(5.10) and (5.7)-(5.9)-(5.10). Equation (5.7) corresponds to the time-continuous equation (4.5), discretized in time using the midpoint scheme.

The equation (5.8) does not correspond to the time continuous equation (4.5), but introduces in the timediscrete setting a strong dissipation (actually of parabolic nature) of the high-frequency components of the solutions, which is needed to prove uniform stabilization properties in the fully discrete setting. If considering (5.9) instead, then the high-frequency components of the solutions are not efficiently damped out by the time-discrete approximation schemes, except if they simply do not exist, which can be guaranteed by imposing a CFL type condition on the discretization parameters.

To be more precise, using [11], we obtain:

Theorem 5.2. Let $M$ be a real number greater than one, and consider a sequence $\left(\mathcal{S}_{n}\right)_{n}$ of $M$-regular meshes, and a sequence of damping functions $\sigma_{n}$ satisfying (4.6).

(1) Then there exist positive constants $C$ and $\mu$ such that for all $n$ and $\Delta t>0$, any solution of (5.7)-(5.8)(5.10) satisfies

$$
E_{n}^{\tau} \leq C E_{n}^{0} \exp (-\mu \tau \Delta t), \quad \tau \in \mathbb{N} .
$$

(2) The same conclusion holds for solutions of (5.7)-(5.9)-(5.10) providing that the quantity $(n+1)^{2} \Delta t$ is bounded when $n \rightarrow \infty, \Delta t \rightarrow 0$.

We emphasize that system (5.7)-(5.9)-(5.10) may be non uniformly exponentially stable with respect to both $\Delta t>0$ and $n$ when no CFL condition is required. In this case, one indeed needs to introduce a numerical viscosity term in the equations to ensure uniform stabilization properties. Note that several other numerical viscosity terms are possible: we refer to [11] for general assumptions on the numerical viscosity term needed in the equations.

\section{Further comments}

In this paper, we have analyzed a space semi-discrete scheme derived from a mixed finite element method for a $1 \mathrm{~d}$ wave equation, which has a good behavior with respect to both stabilization and controllability properties for a large class of nonuniform meshes.

1. The key point of our analysis is the description of the spectrum of the space discrete operator given in Theorems 2.1-2.3. It is particularly surprising that the spectrum can be described in a rather explicit way for any mesh. This does not seem to be the case for other classical schemes, as the ones provided by finite difference or finite element methods. To our knowledge, in these cases, only asymptotic distributions of the eigenvalues are available, see for instance [3] and the literature therein.

2. It would be particularly challenging to understand the behavior of the discrete waves in higher dimension on nonuniform meshes. To our knowledge, this question has not been addressed so far. We expect this question 
to be difficult to address with the tools used until now, which require either a good knowledge of the eigenvalues (see [5,6,17,23-26,31] and our own approach) or the existence of multipliers that behave well (see [10,27,28]) on the discrete systems. This issue is currently under investigation by the author.

3. It would be interesting to estimate the (asymptotic) decay rate for the semi-discrete damped equation as in the continuous case, see [8]. In the continuous case, the computation of the decay rate of the energy is technically involved and requires to work directly on the damped system. We refer to the works $[8,9,20]$ that deal with these questions for damped wave equations.

To our knowledge, even in the case of uniform meshes, this question is still open. Only some partial results in this direction are available in [10] for the space semi-discrete Perfectly Matched Layers equations (see [2]).

Acknowledgements. The author is grateful to E. Zuazua and J.-P. Puel for several suggestions and remarks related to this work.

\section{REFERENCES}

[1] H.T. Banks, K. Ito and C. Wang, Exponentially stable approximations of weakly damped wave equations, in Estimation and control of distributed parameter systems (Vorau, 1990), Internat. Ser. Numer. Math. 100, Birkhäuser, Basel (1991) 1-33.

[2] J.-P. Bérenger, A perfectly matched layer for the absorption of electromagnetic waves. J. Comput. Phys. 114 (1994) 185-200.

[3] E. Bogomolny, O. Bohigas and C. Schmit, Spectral properties of distance matrices. J. Phys. A 36 (2003) 3595-3616.

[4] T.J. Bridges and S. Reich, Numerical methods for Hamiltonian PDEs. J. Phys. A 39 (2006) 5287-5320.

[5] C. Castro and S. Micu, Boundary controllability of a linear semi-discrete 1-d wave equation derived from a mixed finite element method. Numer. Math. 102 (2006) 413-462.

[6] C. Castro, S. Micu and A. Münch, Numerical approximation of the boundary control for the wave equation with mixed finite elements in a square. IMA J. Numer. Anal. 28 (2008) 186-214.

[7] L.C. Cowsar, T.F. Dupont and M.F. Wheeler, A priori estimates for mixed finite element methods for the wave equations. Comput. Methods Appl. Mech. Engrg. 82 (1990) 205-222.

[8] S. Cox and E. Zuazua, The rate at which energy decays in a damped string. Comm. Partial Differ. Equ. 19 (1994) $213-243$.

[9] S. Cox and E. Zuazua, The rate at which energy decays in a string damped at one end. Indiana Univ. Math. J. 44 (1995) 545-573.

[10] S. Ervedoza and E. Zuazua, Perfectly matched layers in 1-d: Energy decay for continuous and semi-discrete waves. Numer. Math. 109 (2008) 597-634.

[11] S. Ervedoza and E. Zuazua, Uniformly exponentially stable approximations for a class of damped systems. J. Math. Pures Appl. (to appear).

[12] S. Ervedoza, C. Zheng and E. Zuazua, On the observability of time-discrete conservative linear systems. J. Funct. Anal. 254 (2008) 3037-3078.

[13] J. Frank, B.E. Moore and S. Reich, Linear PDEs and numerical methods that preserve a multisymplectic conservation law. SIAM J. Sci. Comput. 28 (2006) 260-277 (electronic).

[14] R. Glowinski, Ensuring well-posedness by analogy: Stokes problem and boundary control for the wave equation. J. Comput. Phys. 103 (1992) 189-221.

[15] R. Glowinski, W. Kinton and M.F. Wheeler, A mixed finite element formulation for the boundary controllability of the wave equation. Internat. J. Numer. Methods Engrg. 27 (1989) 623-635.

[16] A. Haraux, Une remarque sur la stabilisation de certains systèmes du deuxième ordre en temps. Portugal. Math. 46 (1989) $245-258$.

[17] J.A. Infante and E. Zuazua, Boundary observability for the space semi discretizations of the 1-d wave equation. Math. Model. Num. Ann. 33 (1999) 407-438.

[18] A.E. Ingham, Some trigonometrical inequalities with applications to the theory of series. Math. Z. 41 (1936) 367-379.

[19] S. Labbé and E. Trélat, Uniform controllability of semidiscrete approximations of parabolic control systems. Systems Control Lett. 55 (2006) 597-609.

[20] G. Lebeau, Équations des ondes amorties, in Séminaire sur les Équations aux Dérivées Partielles, 1993-1994, École Polytechnique, France (1994).

[21] J.-L. Lions, Contrôlabilité exacte, Perturbations et Stabilisation de Systèmes Distribués, Tome 1 : Contrôlabilité exacte, RMA 8. Masson (1988).

[22] F. Macià, The effect of group velocity in the numerical analysis of control problems for the wave equation, in Mathematical and numerical aspects of wave propagation - WAVES 2003, Springer, Berlin (2003) 195-200.

[23] A. Münch, A uniformly controllable and implicit scheme for the 1-D wave equation. ESAIM: M2AN 39 (2005) $377-418$. 
[24] M. Negreanu and E. Zuazua, Convergence of a multigrid method for the controllability of a 1-d wave equation. C. R. Math. Acad. Sci. Paris 338 (2004) 413-418.

[25] M. Negreanu, A.-M. Matache and C. Schwab, Wavelet filtering for exact controllability of the wave equation. SIAM J. Sci. Comput. 28 (2006) 1851-1885 (electronic).

[26] K. Ramdani, T. Takahashi and M. Tucsnak, Uniformly exponentially stable approximations for a class of second order evolution equations - application to LQR problems. ESAIM: COCV 13 (2007) 503-527.

[27] L.R. Tcheugoué Tébou and E. Zuazua, Uniform exponential long time decay for the space semi-discretization of a locally damped wave equation via an artificial numerical viscosity. Numer. Math. 95 (2003) 563-598.

[28] L.R. Tcheugoué Tebou and E. Zuazua, Uniform boundary stabilization of the finite difference space discretization of the 1-d wave equation. Adv. Comput. Math. 26 (2007) 337-365.

[29] L.N. Trefethen, Group velocity in finite difference schemes. SIAM Rev. 24 (1982) 113-136.

[30] R.M. Young, An introduction to nonharmonic Fourier series. Academic Press Inc., San Diego, CA, first edition (2001).

[31] E. Zuazua, Boundary observability for the finite-difference space semi-discretizations of the 2-D wave equation in the square. J. Math. Pures Appl. 78 (1999) 523-563.

[32] E. Zuazua, Propagation, observation, and control of waves approximated by finite difference methods. SIAM Rev. 47 (2005) 197-243 (electronic). 